ACCEPTED MANUSCRIPT

\title{
4D Printed Tunable Mechanical Metamaterials with Shape Memory Operations
}

To cite this article before publication: Mahdi Bodaghi et al 2019 Smart Mater. Struct. in press https://doi.org/10.1088/1361-665X/ab0b6b

\section{Manuscript version: Accepted Manuscript}

Accepted Manuscript is "the version of the article accepted for publication including all changes made as a result of the peer review process, and which may also include the addition to the article by IOP Publishing of a header, an article ID, a cover sheet and/or an 'Accepted Manuscript' watermark, but excluding any other editing, typesetting or other changes made by IOP Publishing and/or its licensors"

This Accepted Manuscript is @ 2019 IOP Publishing Ltd.

During the embargo period (the 12 month period from the publication of the Version of Record of this article), the Accepted Manuscript is fully protected by copyright and cannot be reused or reposted elsewhere.

As the Version of Record of this article is going to be / has been published on a subscription basis, this Accepted Manuscript is available for reuse under a CC BY-NC-ND 3.0 licence after the 12 month embargo period.

After the embargo period, everyone is permitted to use copy and redistribute this article for non-commercial purposes only, provided that they adhere to all the terms of the licence https://creativecommons.org/licences/by-nc-nd/3.0

Although reasonable endeavours have been taken to obtain all necessary permissions from third parties to include their copyrighted content within this article, their full citation and copyright line may not be present in this Accepted Manuscript version. Before using any content from this article, please refer to the Version of Record on IOPscience once published for full citation and copyright details, as permissions will likely be required. All third party content is fully copyright protected, unless specifically stated otherwise in the figure caption in the Version of Record.

View the article online for updates and enhancements. 


\title{
$A B S T R A C T$
}

\section{D Printed Tunable Mechanical Metamaterials with Shape Memory Operations}

\author{
M. Bodaghi ${ }^{1,2}$ and W. H. Liao ${ }^{1 \dagger}$ \\ ${ }^{1}$ Smart Materials and Structures Laboratory, Department of Mechanical and Automation Engineering, \\ The Chinese University of Hong Kong, Shatin, N.T., Hong Kong, China \\ ${ }^{2}$ Department of Engineering, School of Science and Technology, Nottingham Trent University, United Kingdom
}

The aim of this paper is to introduce tunable continuous-stable metamaterials with reversible thermomechanical memory operations by four-dimensional (4D) printing technology. They are developed based on an understanding on glassy-rubbery behaviors of shape memory polymers and hot/cold programming derived from experiments and theory. Fused decomposition modeling as a well-known 3D printing technology is implemented to fabricate mechanical metamaterials. They are experimentally tested revealing elastic-plastic and hyper-elastic behaviors in low and high temperatures at a large deformation range. A computational design tool is developed by implementing a 3D phenomenological constitutive model coupled with a geometrically non-linear finite element method. Governing equations are then solved by an elastic-predictor plastic-corrector return map procedure along with the Newton-Raphson and Riks techniques to trace non-linear equilibrium path. A tunable reversible mechanical metamaterial unit with bistable memory operations is printed and tested experimentally and numerically. By a combination of cold and hot programming, the unit shows potential applications in mimicking electronic memory devices like tactile displays and designing surface adaptive structures. Another design of the unit shows potentials to serve in designing self-deployable bio-medical stents. Experiments are also conducted to demonstrate potential applications of cold programming for introducing recoverable rolling-up chiral metamaterials and load-resistance supportive auxetics.

Keywords: 4D printing, SMPs, Tunable Metamaterials, Memory Operations, Experiments, FEM

Correspónding Author. Tel.: +852 3943 8341; fax: +852 26036002.

E-mail address: whliao@cuhk.edu.hk 


\section{Introduction}

Mechanical metamaterials have been introduced to realize unique mechanical features that are not found in nature. They have shown interesting and unconventional phenomena such as zero shear modulus [1], zero/negative Poisson's ratio [2-9], variable softening/hardening [9-11], multistability [12-14] and self-folding features [15-21]. Most mechanical metamaterials derive their properties from their structure and programmed architecture rather than the bulk behavior of their constituents.

In recent years, additive manufacturing technologies have enabled fabrication of mechanical metamaterials. For instance, Ren et al. [7] introduced 3D printed tubular metamaterial structures like stents which exhibited auxetic behavior in both compression and tension, for the first time. $\mathrm{Li}$ et al. [8] investigated compressive behaviors of the 3D printed auxetics reinforced composites and achieved significant enhancement of their stiffness and energy absorption due to the negative Poisson's ratio effect of the auxetic reinforcements. Bodaghi et al. [9] investigated softening and hardening behaviors of $3 \mathrm{D}$ printed lattice-based mechanical metamaterials under tensile and compressive loading. Numerical and experimental results revealed that anisotropic metamaterial responses significantly depend on unit-cell shape, type and magnitude of mechanical loading. Chen et al. [10] reported a class of 3D printed lattice metamaterials with mechanically tunable negative Poisson's ratios and vibration-mitigation capability. Their experimental and numerical results indicated that the proposed lattices exhibited extreme Poisson's-ratio variations between 0.7 and 0.5 over large tensile deformations up to $50 \%$. Hedayati et al. [11] proposed action-at-adistance $3 \mathrm{D}$ printed mechanical metamaterials by grading designs of auxetic and conventional unit cells with changing Poisson's ratios. Rafsanjani et al. [13] proposed a multi-stable 2-D mechanical metamaterial comprising a periodic arrangement of snapping units with tunable tensile behavior. 
The unit cell geometry of the metamaterial was composed of load bearing and snapping segments and fabricated by selective laser sintering 3D printing technology. Che et al. [14] presented approaches of small geometric variations to tune the deformation sequence of 2D multi-stable mechanical metamaterial fabricated by PolyJet 3D printing technology. The designed metamaterial remains in a deformed configuration even after removal of the pre-load due to multi-stability. While 3D printing of traditional materials leads to static 3D mechanical metamaterials, 4D printing of responsive materials adds the fourth dimension as it leads to adaptive metamaterials that, with an appropriate stimulus, change their shape over time. For example, Bodaghi et al. [19, 20] showed how fused decomposition modeling (FDM) as a filament-based 3D printing technology can engineer adaptive mechanical metamaterials with performance-driven functionality built directly into materials. The developed metamaterials showed dual/triple shape memory features when heated. By developing internal compressive stress during PolyJet 3D printing process, Ding et al. [21] introduced 1D composite rod architectures that self-assembled upon heating and then retained the 3D configuration when cooled. Using multi-material inkjet PolyJet 3D printing technology, Akbari et al. [22] introduced shape memory structures including a morphing wing flap and a deployable structure which consist of active and flexible hinges joining rigid parts.

The mechanical metamaterials with mono/multi-stable configurations and/or self-folding features reviewed above just show one-way actuatory functions. However, reversible thermomechanical memory operations could be beneficial not only to mimic optical/electronic memory devices, but also to tune and store the flow of mechanical energy for actuation purposes.

The main objective of this paper is to develop tunable continuous-stable metamaterials with reversible thermo-mechanical memory operations by 4D FDM printing. The main methodology is based on arranging geometrical configuration and hot-cold programming of shape memory 
polymers (SMPs) to introduce reversible shape adaptive structures. It is experimentally revealed that the printed SMPs behave elasto-plastically and hyper-elastically respectively at glassy and rubbery phases. In order to enable design of tunable shape-memory mechanical metamaterials, a 3D macroscopic constitutive model is developed. It has capability of replicating elasto-plastic, hyper-elastic, hot and cold programming. A finite element method (FEM) based on the non-linear Green-Lagrange strains is implemented and then solved via an elastic-predictor plastic-corrector return map procedure accomplished by iterative incremental Newton-Raphson and Riks techniques. The FDM printing technology and FEM solution are implemented to digitally design and fabricate adaptive metamaterials with reversible shape memory features. It is shown experimentally and numerically that the printed metamaterials have great potentials in mimicking electronic memory devices like tactile displays and designing surface adaptive structures and selfdeployable bio-medical stents [7]. Experiments are also carried out to introduce rolling-up chiral metamaterials and load-resistance supportive auxetics by cold programming method. Due to the absence of similar concept and results in the specialized literature, this paper is likely to advance the state of the art tunable mechanical metamaterials with reversible shape memory features.

\section{Materials and Methodology}

The tunable mechanical metamaterials with reversible shape memory operations are developed based on an understanding on 3D printing technology and thermo-mechanical behaviors of SMP materials derived from basic experiments and theory which are presented in this section. 


\subsection{Materials and Fabrication}

The FDM 3D printer used in this work is New Creator Pro from Flash-Forge. It is fed by polyurethane-based SMP filaments with a diameter of $1.75 \mathrm{~mm}$ and glass transition temperature of $55{ }^{\circ} \mathrm{C}$ (SMP Technologies Inc., Tokyo, Japan). The filament is heated by the liquefier accomplished with heaters and extruded through a nozzle tip with physical diameter of $0.4 \mathrm{~mm}$. CraftWare software is utilized to convert digital 3D object designed by SolidWorks and exported as a stereolithography (.stl) file into G-code toolpath format. The infill percentage is considered to be $100 \%$ in order to fabricate solid objects of high density and other printer settings are as follows: raster angle $\left(0^{\circ}\right)$; temperature of the liquefier, build tray and chamber $\left(230,23,23{ }^{\circ} \mathrm{C}\right)$; speed while extruding $(10 \mathrm{~mm} / \mathrm{s})$; speed while traveling $(150 \mathrm{~mm} / \mathrm{s})$; number of shells $(2)$; and layer height $(0.2$ $\mathrm{mm}$ ). Printrun software is also implemented to handle the $3 \mathrm{D}$ printing procedure.

Next, elastic thermo-mechanical/properties of the SMP printed materials such as Young's modulus, glass transition temperature, and the coefficient of thermal expansion are measured. Samples with dimension of $16 \times 1.6 \times 1 \mathrm{~mm}$ are printed and analyzed via a dynamic-mechanical analyzer (DMA) (Model 242, NETZSCH) in tension and axial modes. Three specimens are printed and filled with a perimeter raster in which the print raster is parallel to the length of the gauge section $\left(0^{\circ}\right.$ raster angle in the axial gauge section). Furthermore, five repetition tests are performed on each specimen in the axial mode along the printing direction with frequency of force oscillation $1 \mathrm{~Hz}$ and heating ramp of $150^{\circ} \mathrm{C} / \mathrm{min}$ increasing from -20 to $85^{\circ} \mathrm{C}$. Arithmetic mean of all values is reported as an average value. In this respect, the testing results of all the specimens are considered due to close agreement. DMA results in terms of Young's modulus, $E$, storage modulus, $E_{s}$, and phase lag, $\tan (\delta)$, are illustrated in Fig. 1. As it can be seen, the glassy phase of the 3D printed SMP at $T_{l}=23{ }^{\circ} \mathrm{C}$ with storage modulus of $1.66 \mathrm{GPa}$ completely transforms to rubbery 
phase with storage modulus of $3.18 \mathrm{MPa}$ in the temperature of $T_{h}=85^{\circ} \mathrm{C}$. The phase lag peaks at $61{ }^{\circ} \mathrm{C}$ during SMP phase transformation that is assumed as glass transition temperature so-called $T_{g}$

Thermo-mechanical properties of the 3D printed SMP at glassy and rubbery phases in terms of elastic modulus $(E)$, Poisson's ratio (v) and thermal expansion coefficient $(\alpha)$ are measured by tensile and thermal strain tests. Tests are carried out at 23 and $85{ }^{\circ} C$. Three specimens for each sample are printed $\left(0^{\circ}\right.$ raster angle in the axial gauge section) based on the geometry and dimensions set by the ASTM D638 standard [23]. Tinius Olsen ${ }^{\circledR} H 5 k 5$ tensile machine (Horsham, PA, USA) with a $5 \mathrm{kN}$ load-cell and an attached home-made thermal chamber is employed to perform uniaxial mechanical tests at constant speed of $1 \mathrm{~mm} / \mathrm{min}$. Five repetition tests are performed on each specimen. All stress and strain values are computed using initial cross-sectional area and length of the test specimens in the calculations. For each series of tests, arithmetic mean of all values is reported as an average value. The testing results of all the specimens are also taken into account due to close agreement. Average elastic modulus, Poisson's ratio and thermal expansion coefficient are measured as $E_{g}=1.66 \mathrm{GPa}, E_{r}=3.3 \mathrm{MPa} ; \mathrm{v}_{g}=0.35, \mathrm{v}_{r}=0.44 ; \alpha_{g}=\alpha_{r}$ $=0.00011 / K$ where sub-scripts ' $g$ ' and ' $r$ ' signify glassy and rubbery phases here and henceforth. It can be concluded that, while thermal expansion coefficient is temperature-independent, the SMP phase transformation increases Poisson's ratio up to $26 \%$ and makes the material nearly incompressible.

Next, mechanical behaviors of the printed SMP in the large strain domain are investigated via printing five specimens and performing tensile tests up to $40 \%$ strain followed by unloading at temperatures of 23 and $85^{\circ} \mathrm{C}$. Arithmetic mean of all values is reported as an average value. The testing results of all the specimens are also considered due to close correlation. Engineering stress- 


\subsection{SMP Constitutive Môdeling}

Classical constitutive models like elastic-plastic and hyper-elastic models can independently be implemented to replicate mechanical behaviors of polymers at glassy and rubbery phases. However, to simulate coupled hot and cold programming of polymers with reversible shape recovery features, a standalone 3D phenomenological model originally proposed by Bodaghi et al. [20] is adopted here. It is developed within the framework of continuum thermodynamics with internal variables in the large strain regime. 


\subsubsection{Basic assumptions}

The volume fraction of the glassy and rubbery phases, denoted by $v_{g}$ and $v_{r}$, are considered as scalar variables. As their summation should meet unity, $v_{g}+v_{r}=1$, there is just one independent volume fraction via $v_{r}=1-v_{g}$. The glassy-phase volume fraction is assumed to be temperaturedependent as a generally well-known assumption, i.e., $v_{g}=v_{g}(T)$. The rubbery and glassy phases in SMPs are assumed to be linked to each other in a parallel manner. It is formulated as:

$\boldsymbol{F}=\boldsymbol{F}_{g t}=\boldsymbol{F}_{r t}$

where $\boldsymbol{F}$ denotes the total deformation gradient. Considering definition of the right Cauchy-Green deformation tensor as $\boldsymbol{C}=\boldsymbol{F}^{T} \boldsymbol{F}$, one obtains:

$\boldsymbol{C}=\boldsymbol{C}_{g t}=\boldsymbol{C}_{r t}$

The total deformation gradient of the glassy phase $\boldsymbol{F}_{g t}$ is then considered to be a combination of active glassy phase contribution, $\boldsymbol{F}_{g a}$, and a frozen contribution, $\boldsymbol{F}_{g f}$ that can be separated by a multiplicative decomposition scheme. It is stated as:

$\boldsymbol{F}_{g t}=\boldsymbol{F}_{g a} \boldsymbol{F}_{g f}$

$\boldsymbol{F}_{g f}$ indicates deformation induced by hot programming that can be stored temporarily at low temperature and released upon heating over the transition temperature. The SMP behavior through cold programming is assumed to be elasto-plastic. Therefore, a multiplicative decomposition of active glassy phase deformation gradient $\boldsymbol{F}_{g a}$ into an elastic part $\boldsymbol{F}_{g e}$, characterized with respect to a middle configuration, and a plastic part $\boldsymbol{F}_{g p}$ associated to the reference configuration is considered as: 
$\boldsymbol{F}_{g a}=\boldsymbol{F}_{g e} \boldsymbol{F}_{g p}$

The rubbery phase behavior is assumed to be hyper-elastic. Therefore, $\boldsymbol{F}_{g t}$ coincides with the hyper-elastic part as:

$\boldsymbol{F}_{r t}=\boldsymbol{F}_{r e}$

Considering Green deformation tensor definition, one obtains:

$\boldsymbol{C}=\boldsymbol{F}_{g f}^{T} \boldsymbol{F}_{g p}^{T} \boldsymbol{F}_{g e}^{T} \boldsymbol{F}_{g e} \boldsymbol{F}_{g p} \boldsymbol{F}_{g f}$

Consequently, elastic Green deformation tensor of the glassy phase can be written in terms of Green deformation tensor as:

$\boldsymbol{C}_{g e}=\boldsymbol{F}_{g p}^{-T} \boldsymbol{F}_{g f}^{-T} \boldsymbol{C} \boldsymbol{F}_{g f}^{-1} \boldsymbol{F}_{g p}^{-1}$

Elastic Green deformation tensor of the rubbery phase can also be considered as:

$\boldsymbol{C}_{r e}=\boldsymbol{C}$

\subsubsection{Model derivation}

In order to derive SMP constitutive equations, the Green deformation tensor $\boldsymbol{C}$ and the absolute temperature $T$ are chosen as external variables. Furthermore, $\boldsymbol{C}_{g p}$ defined as $\boldsymbol{F}_{g p}^{T} \boldsymbol{F}_{g p}$ is considered as internal variable. Saint-Venant-Kirchhoff and Mooney-Rivlin strain energy functions are adopted for glassy and rubbery phases. Kinematic-type hardening is also assumed for the plastic contribution of the glassy phase [20]. Implementing the second law of thermodynamics in the framework of Clausius-Duhem inequality [24], constitutive equations can be derived as:

I) Stress field: 


$$
\boldsymbol{S}=v_{g} \boldsymbol{S}_{g}+\left(1-v_{g}\right) \boldsymbol{S}_{r}
$$

in which $S_{g}$ and $S_{r}$ are glassy and rubbery components of the second Piola-Kirchhoff stress tensor defined by a rule of mixtures. They can be expressed as:

$$
\begin{aligned}
& \boldsymbol{S}_{g}=\left(\left(\frac{1}{2} \lambda_{g}+\mu_{g}\right)\left(I_{1 g e}-3\right)+2 \mu_{g}\right) \boldsymbol{C}_{g p f}^{-1}-\mu_{g}\left(I_{1 g e} \boldsymbol{I}-\boldsymbol{C}_{g p f}^{-1} \boldsymbol{C}\right) \boldsymbol{C}_{g p f}^{-1}-3 \alpha_{g} \kappa_{g}\left(T-T_{0}\right) \boldsymbol{I} \\
& \boldsymbol{S}_{r}=2\left(c_{1 r}+c_{2 r} I_{1 r}\right) \boldsymbol{I}-2 c_{2} \boldsymbol{C}-\frac{2}{3}\left(c_{1 r} I_{1 r}+2 c_{2 r} I_{2 r}\right) \boldsymbol{C}^{-1}
\end{aligned}
$$

where

$$
\boldsymbol{C}_{g p f}=\boldsymbol{F}_{g f} \boldsymbol{C}_{g p} \boldsymbol{F}_{g f}^{T}
$$

and, $I_{1}$ and $I_{2}$ are the first and second invariant of the Green deformation tensor. Also, $\lambda$ and $\mu$ signify Lamè constants, while $c_{1}$ and $c_{2}$ are material constants related to the distortional response of the rubbery phase. Furthermore, $\alpha$ and $\kappa$ denote thermal expansion coefficient and bulk modulus. II) Mechanism of cold programming:

a) Thermo-dynamical dissipative force

$$
\boldsymbol{T}_{g p}=\left(\left(\frac{1}{4} \lambda_{g}+\frac{1}{2} \mu_{g}\right)\left(I_{1 g e}-3\right)+\mu_{g}\right) \boldsymbol{C}_{g p}^{-1} \boldsymbol{C}_{t f} \boldsymbol{C}_{g p}^{-1}-\frac{1}{2} \mu_{g}\left(I_{1 g e}-\boldsymbol{C}_{g p}^{-1} \boldsymbol{C}_{t f}\right) \boldsymbol{C}_{g p}^{-1} \boldsymbol{C}_{t f} \boldsymbol{C}_{g p}^{-1}-\frac{1}{4} \psi\left(\boldsymbol{C}_{g p}-\boldsymbol{I}\right)
$$

where

$$
\boldsymbol{C}_{t f}=\boldsymbol{F}_{g f}^{-T} \boldsymbol{C F}_{g f}^{-1}
$$

and $\psi$ is a stress-like material parameter that controls initial hardening during glassy-phase plastic evolution.

b) Flow rule

$$
\dot{\boldsymbol{C}}_{g p}=\dot{\eta} \boldsymbol{T}_{g p}^{D} /\left\|\boldsymbol{T}_{g p}^{D}\right\|
$$


where $\eta$ is non-negative consistency coefficient. The superscript $D$ indicates deviatoric component of $\boldsymbol{T}_{g p}$ that has been assumed to contribute in the plastic evolution.

\section{c) Limit function}

$$
L_{g p}=\left\|\boldsymbol{T}_{g p}^{D}\right\|-Y_{g p}
$$

where $Y_{g p}$ is a constant threshold value to activate mechanically induced plastic deformation. The evolution of $\boldsymbol{C}_{g p}$ is finally constrained by

\section{d) Kuhn-Tucker conditions}

$$
L_{g p} \leq 0, \dot{\eta} \geq 0, L_{g p} \dot{\eta}=0
$$

Plasticity grows inside the glassy phase through mechanical loading at low temperature $\left(T \leq T_{l}\right)$ or during cooling in the SMP transition range $\left(T_{l}<T<T_{h}\right)$. It is recovered by simply heating within transition domain and will disappear beyond $T_{h}$. Therefore, the plastic deformation evolution needs to be restricted mathematically as:

e) Thermal conditions

$$
\left\{\begin{array}{l}
\text { if }\left(T \leq T_{l}\right) \text { or }\left(T_{l}<T<T_{h}, \dot{T} \leq 0\right) \text { then } C_{g p} \text { evolves via Eq. (27) } \\
\text { if }\left(T_{l}<T<T_{h}, \dot{T} \geq 0\right) \text { then } C_{g p} \text { remains constant } \\
\text { if }\left(T \geq T_{h}\right) C_{g p} \text { is set } I
\end{array}\right.
$$

\section{III) Mechanism of hot programming:}

The frozen deformation gradient is evolved in the glassy phase as:

$$
\left\{\begin{array}{l}
\text { if }\left(T \geq T_{h}\right) \text { then } \boldsymbol{F}_{g f}=c_{g f}(\boldsymbol{F}-\boldsymbol{I})+\boldsymbol{I} \\
\text { otherwise } \boldsymbol{F}_{g f} \text { remains constant }
\end{array}\right.
$$


Where $c_{f g}$ is a material constant that ranges in $\left[\begin{array}{ll}0 & 1\end{array}\right]$ and sets the shape-fixing ratio in the hotprogramming procedure. When $c_{f g}$ is assumed to be 1 , all the deformation is frozen via $\boldsymbol{F}_{g f}=\boldsymbol{F}$, while setting less than 1 results in imperfect or null shape-fixing.

IV) Mechanism of SMP phase transformation:

$$
v_{g}=-\frac{\tanh \left(a_{1} T_{g}-a_{2} T\right)-\tanh \left(a_{1} T_{g}-a_{2} T_{h}\right)}{\tanh \left(a_{1} T_{g}-a_{2} T_{h}\right)-\tanh \left(a_{1} T_{g}-a_{2} T_{l}\right)}
$$

where $a_{1}$ and $a_{2}$ are set to fit the storage modulus extracted from experimental DMA data.

\subsubsection{Solution method}

The solution of the constitutive model with evolutive cold-programming process is obtained by implementing an elastic-predictor inelastic-corrector return map algorithm details of which can be found in [25]. The plastic deformation is first assumed to remain constant and the limit function, $L_{g p}$, is calculated to examine the admissibility of the trial state. If the plasticity criterion is fulfilled, that is, $L_{g p}>0$, then the trial state of $\boldsymbol{C}_{g p}$ is assumed to be elastic and admissible. However, if $L_{g p} \leq 0$, the Kuhn-Tucker conditions are violated and the trial state lies outside the plasticity surface. In the latter case, the glassy-phase plastic deformation, $\boldsymbol{C}_{g p}$, should be updated. This can be performed by discretizing Eq. (14) via the explicit forward-Euler integration rule and solving non-linear system of algebraic equations through iterative Newton-Raphson scheme [25]. 


\subsection{Finite Element Modelling}

This section is dedicated to establish an FE computational model to digitally design mechanical metamaterials with reversible memory operations. The total Lagrangian description and the principle of minimum total potential energy are employed to formulate equilibrium equations of the 4D printed mechanical metamaterials.

While the SMP model has been developed in a tensorial scheme, the FE model is derived here in the framework of a vectorial notation, which is convenient for numerical implementations. Thus, stress-strain equation (9) can be rewritten, by means of some algebraic manipulation, in a matrixvector layout as:

$$
\boldsymbol{s}=\boldsymbol{E}\left(\boldsymbol{F}_{g f}, \boldsymbol{C}_{g p}, T\right)\left(\boldsymbol{e}-\boldsymbol{q}\left(\boldsymbol{F}_{g f}, \boldsymbol{C}_{g p}, T\right)\right)
$$

where $\boldsymbol{s}$ and $\boldsymbol{e}$ indicate the second Piola-Kirchhoff stress and Green-Lagrange strain vectors. Also, $\boldsymbol{Q}$ and $\boldsymbol{q}$ presents matrix and vector being functions of temperature and components of $\boldsymbol{F}_{g f}$ and $\boldsymbol{C}_{g p}$.

Deformation of the mechanical metamaterial structures is described based on the non-linear Green-Lagrange strain-displacement kinematic expressions as:

$$
\boldsymbol{e}=\left(\boldsymbol{D}_{L}+\frac{1}{2} \boldsymbol{D}_{N}\right) \boldsymbol{u}
$$

where $\boldsymbol{u}$ is displacement field vector defined as:

$$
\boldsymbol{u}=\left\{\begin{array}{lll}
u_{X} & u_{Y} & u_{Z}
\end{array}\right\}^{T}
$$

in which $u_{i}(i=X, Y, Z)$ are displacements along $X, Y, Z$ coordinates, respectively. $\boldsymbol{D}_{L}$ and $\boldsymbol{D}_{N}$ are also linear and non-linear differential operator matrices. Signifying partial differentiation with respect to the spatial coordinates $X, Y, Z$ by: 


$$
\frac{\partial}{\partial X}=\partial_{X}, \frac{\partial}{\partial Y}=\partial_{Y}, \frac{\partial}{\partial Z}=\partial_{Z}
$$

the differential operator matrices are defined by:

$$
\boldsymbol{D}_{L}=\left[\begin{array}{ccc}
\partial_{X} & 0 & 0 \\
0 & \partial_{Y} & 0 \\
0 & 0 & \partial_{Z} \\
0 & \partial_{Z} & \partial_{Y} \\
\partial_{Z} & 0 & \partial_{X} \\
\partial_{Y} & \partial_{X} & 0
\end{array}\right], \boldsymbol{D}_{N}=\left[\begin{array}{ccc}
\partial_{X} u_{X} \partial_{X} & \partial_{X} u_{Y} \partial_{X} & \partial_{X} u_{Z} \partial_{X} \\
\partial_{Y} u_{X} \partial_{Y} & \partial_{Y} u_{Y} \partial_{Y} & \partial_{Y} u_{Z} \partial_{Y} \\
\partial_{Z} u_{X} \partial_{Z} & \partial_{Z} u_{Y} \partial_{Z} & \partial_{Z} u_{Z} \partial_{Z} \\
\partial_{Y} u_{X} \partial_{Z}+\partial_{Z} u_{X} \partial_{Y} & \partial_{Y} u_{Y} \partial_{Z}+\partial_{Z} u_{Y} \partial_{Y} & \partial_{2} u_{Z} \partial_{Z}+\partial_{Z} u_{Z} \partial_{Y} \\
\partial_{X} u_{X} \partial_{Z}+\partial_{Z} u_{X} \partial_{X} & \partial_{X} u_{Y} \partial_{Z}+\partial_{Z} u_{Y} \partial_{X} & \partial_{X} u_{Z} \partial_{Z}+\partial_{Z} u_{Z} \partial_{X} \\
\partial_{X} u_{X} \partial_{Y}+\partial_{Y} u_{X} \partial_{X} & \partial_{X} u_{Y} \partial_{Y}+\partial_{Y} u_{Y} \partial_{X} & \partial_{X} u_{Z} \partial_{Y}+\partial_{Y} u_{Z} \partial_{X}
\end{array}\right]
$$

Next, the principle of minimum total potential energy is implemented to extract the governing equations of equilibrium and associated boundary conditions for the 4D printed mechanical metamaterials. It is stated as:

$$
\delta \Pi=\delta U-\delta W=0
$$

where $\delta$ is the variational operator, while $\delta \Pi, \delta U$ and $\delta W$ present variation of total potential energy, of strain energy, and of work performed by applied loads, respectively.

The internal virtual work stored in the structure can be expressed as:

$$
\delta U=\int_{V} \delta \boldsymbol{e}^{T} \boldsymbol{s} d V
$$

where $V$ indicates the structure volume. Considering the stress definition (20), it can be rewritten as:

$$
\delta U=\int_{V} \delta \boldsymbol{e}^{T} \boldsymbol{E}(\boldsymbol{e}-\boldsymbol{q}) d V
$$

The external virtual work done by applied loads $\boldsymbol{l}$ in moving through the virtual displacement $\delta \boldsymbol{u}$ is also expressed as:

$$
\delta W=\delta \boldsymbol{u}^{T} \boldsymbol{l}
$$

in which 


$$
\boldsymbol{l}=\left\{\begin{array}{lll}
l_{X} & l_{Y} & l_{Z}
\end{array}\right\}^{T}
$$

symbolizes concentrated forces applied along $X, Y, Z$ coordinates.

In order to find a solution for the present problem, a Ritz-based FE method is engaged. To this end, a 3D twenty-node serendipity hexahedron element is employed for the domain discretization, due to its good approximation property. The element has eight corner nodes and twelve nodes along the edges. Each node also has three degrees of freedom including displacements along $X, Y$, $Z$ coordinates. Assuming quadrilateral shape functions $\Theta_{i}(X, Y, Z)(i=1 . .20)$, details of which can be found in [26], the displacement field vector $\boldsymbol{u}$ can be interpolated in terms of mechanical nodal variables, $u_{i-j}(i=X, Y, Z ; j=1 \ldots 20)$, as:

$$
\boldsymbol{u}=\boldsymbol{\Theta n}
$$

where shape-function matrix $\boldsymbol{\Theta}$ and nodal displacement vector $\boldsymbol{n}$ are defined as:

$$
\begin{aligned}
& \boldsymbol{\Theta}=\left[\begin{array}{ccccccc}
\Theta_{1} & 0 & 0 & \cdots & \Theta_{20} & 0 & 0 \\
0 & \Theta_{1} & 0 & \cdots & 0 & \Theta_{20} & 0 \\
0 & 0 & \Theta_{1} & \cdots & 0 & 0 & \Theta_{20}
\end{array}\right] \\
& \boldsymbol{n}=\left\{\begin{array}{llllll}
u_{X-1} & u_{Y-1} & u_{Z-1} & \cdots & u_{X-20} & u_{Y-20} \\
u_{Z-20}
\end{array}\right\}^{T}
\end{aligned}
$$

The discretized form of the virtual displacement field becomes:

$$
\delta \boldsymbol{u}=\boldsymbol{\Theta} \delta \boldsymbol{n}
$$

By means of Eq. (30), the strain field (21) can be interpolated as:

$$
\boldsymbol{e}=\left(\boldsymbol{D}_{L}+\frac{1}{2} \overline{\boldsymbol{D}}_{N}\right) \boldsymbol{\Theta n}
$$

where the differential operator matrix of $\overline{\boldsymbol{D}}_{N}$ is a function of nodal displacements due to the geometric non-linearity expressed as: 


$$
\overline{\boldsymbol{D}}_{N}=\left[\begin{array}{ccc}
\partial_{X} \bar{u}_{X} \partial_{X} & \partial_{X} \bar{u}_{Y} \partial_{X} & \partial_{X} \bar{u}_{Z} \partial_{X} \\
\partial_{Y} \bar{u}_{X} \partial_{Y} & \partial_{Y} \bar{u}_{Y} \partial_{Y} & \partial_{Y} \bar{u}_{Z} \partial_{Y} \\
\partial_{Z} \bar{u}_{X} \partial_{Z} & \partial_{Z} \bar{u}_{Y} \partial_{Z} & \partial_{Z} \bar{u}_{Z} \partial_{Z} \\
\partial_{Y} \bar{u}_{X} \partial_{Z}+\partial_{Z} \bar{u}_{X} \partial_{Y} & \partial_{Y} \bar{u}_{Y} \partial_{Z}+\partial_{Z} \bar{u}_{Y} \partial_{Y} & \partial_{2} \bar{u}_{Z} \partial_{Z}+\partial_{Z} \bar{u}_{Z} \partial_{Y} \\
\partial_{X} \bar{u}_{X} \partial_{Z}+\partial_{Z} \bar{u}_{X} \partial_{X} & \partial_{X} \bar{u}_{Y} \partial_{Z}+\partial_{Z} \bar{u}_{Y} \partial_{X} & \partial_{X} \bar{u}_{Z} \partial_{Z}+\partial_{Z} \bar{u}_{Z} \partial_{X} \\
\partial_{X} \bar{u}_{X} \partial_{Y}+\partial_{Y} \bar{u}_{X} \partial_{X} & \partial_{X} \bar{u}_{Y} \partial_{Y}+\partial_{Y} \bar{u}_{Y} \partial_{X} & \partial_{X} \bar{u}_{Z} \partial_{Y}+\partial_{Y} \bar{u}_{Z} \partial_{X}
\end{array}\right]
$$

in which

$$
\bar{u}_{i}=\sum_{j=1}^{20} u_{i-j} \Theta_{j} \quad, \quad i=X, Y, Z
$$

It can also be proved the variational form of the strain field can be given by:

$$
\delta \boldsymbol{e}=\left(\boldsymbol{D}_{L}+\overline{\boldsymbol{D}}_{N}\right) \boldsymbol{\Theta} \delta \boldsymbol{n}
$$

By substituting the discretized virtual displacement field (32), the strain field (33) and its variation (36) into the virtual energies (27) and (28), and the subsequent results into the principle of minimum total potential energy (25), the governing equation for a parent element is derived as:

$$
\left(\boldsymbol{K}_{0}+\boldsymbol{K}_{L}(\boldsymbol{n})+\boldsymbol{K}_{N}(\boldsymbol{n})\right) \boldsymbol{n}=\boldsymbol{l}_{m}+\boldsymbol{l}_{g p f}
$$

where

$$
\begin{aligned}
& \boldsymbol{K}_{0}=\iiint_{\bar{V}}\left(\boldsymbol{D}_{L} \boldsymbol{\Theta}\right)^{T} \boldsymbol{E}\left(\boldsymbol{D}_{L} \boldsymbol{\Theta}\right)^{T} d \bar{V} \\
& \boldsymbol{K}_{L}=\iiint_{\bar{V}}\left(\frac{1}{2}\left(\boldsymbol{D}_{L} \boldsymbol{\Theta}\right)^{T} \boldsymbol{E}\left(\boldsymbol{D}_{N} \boldsymbol{\Theta}\right)^{T}+\left(\boldsymbol{D}_{N} \boldsymbol{\Theta}\right)^{T} \boldsymbol{E}\left(\boldsymbol{D}_{L} \boldsymbol{\Theta}\right)^{T}\right) d \bar{V} \\
& \boldsymbol{K}_{N}=\iiint_{\bar{V}} \frac{1}{2}\left(\boldsymbol{D}_{N} \boldsymbol{\Theta}\right)^{T} \boldsymbol{E}\left(\boldsymbol{D}_{N} \boldsymbol{\Theta}\right)^{T} d \bar{V} \\
& \boldsymbol{l}_{m}=\left\{\begin{array}{c}
\boldsymbol{l}_{1} \\
\vdots \\
\boldsymbol{l}_{20}
\end{array}\right\}, \quad, \quad \boldsymbol{l}_{i}=\left\{\begin{array}{l}
l_{X-i} \\
l_{Y-i} \\
l_{\mathrm{Z}_{-i}}
\end{array}\right\}, \quad i=1 \ldots 20 \\
& \boldsymbol{l}_{g p f}=\iiint_{\bar{V}}\left(\left(\boldsymbol{D}_{L} \boldsymbol{\Theta}\right)^{T}+\left(\boldsymbol{D}_{N} \boldsymbol{\Theta}\right)^{T}\right) \boldsymbol{E} \boldsymbol{q} d \bar{V}
\end{aligned}
$$

where $\bar{V}$ indicates the element volume. Also, $\boldsymbol{K}_{0}$ is the constant stiffness matrix while $\boldsymbol{K}_{L}$ and $\boldsymbol{K}_{N}$ represent linear and quadratic stiffness matrices, which are functions of nodal displacements, 
respectively. $l_{X-i}, l_{Y-i}$ and $l_{Z-i}$ signify point forces imposed on the $i^{\text {th }}$ node of the parent element along $X, Y$ and $Z$ coordinates, respectively. $\boldsymbol{l}_{g p f}$ is the load vector produced by thermal loading due to temperature change from a reference temperature and/or SMP phase transformation during cold/hot programming. Being $\boldsymbol{q}$ in $\boldsymbol{l}_{g p f}$ as a function of unknown variables of $\boldsymbol{F}_{g f}$ and $\boldsymbol{C}_{g p}$ couples the elemental governing equations of the printed object with SMP constitutive model developed in Section 2.3. Thus, $\boldsymbol{F}_{g f}$ and $\boldsymbol{C}_{g p}$ are held into the volumetric integral of $\boldsymbol{l}_{g p f}$. In this work, temperature field, $T$, is given to be constant through the element volume. Furthermore, $\boldsymbol{F}_{g f}$ and $\boldsymbol{C}_{g p}$ are discretized inside the element by means of 3D Gauss-Legendre quadrature integration approach. Therefore, $\boldsymbol{l}_{g p f}$ is numerically integrated by a $3 \times 3 \times 3$ discrete grid Gauss points involving 3 points along $X, Y$ and $Z$ coordinates.

Finally, Eq. (37) derived for a parent element is utilized to construct global finite element governing equations for the 3D printed mechanical metamaterials by assembly method and applying appropriate boundary conditions. It results in:

$$
\left(\overline{\boldsymbol{K}}_{0}+\overline{\boldsymbol{K}}_{L}+\overline{\boldsymbol{K}}_{N}\right) \overline{\boldsymbol{n}}=\overline{\boldsymbol{l}}_{m}+\overline{\boldsymbol{l}}_{g p f}
$$

This is a non-linear system of equations in terms of unknown nodal displacements, $\bar{n}$, and unknown material variables of $\boldsymbol{F}_{g f}$ and $\boldsymbol{C}_{g p}$ distributed through SMP elements. $\boldsymbol{F}_{g f}$ and $\boldsymbol{C}_{g p}$ need to be calculated by SMP constitutive model while it depends on the unknown strain/displacement field that couples them together. The current problem with both material and geometrical non-linearities is numerically solved by an iterative incremental approach on the basis of the elastic-predictor inelastic-corrector return mapping process. It is accompanied by the 
Newton-Raphson technique to detect non-linear equilibrium path during large deformations details of which can be found elsewhere [26].

\section{4D Printed Mechanical Metamaterials}

In this section, thirteen material properties of the constitutive SMP are first calibrated. Then, some mechanical metamaterials with shape memory operations fabricated by $3 \mathrm{D}$ printing technology are introduced. It is shown that they have great potential in mechanical/electrical/biomedical applications. Using the identified material properties, the FEM tool developed in the previous section is utilized to replicate experimental results.

The parameters of $a_{1}, a_{2}$ and $T_{l}, T_{h}$ introduced in Eq. (19) are determined by means of DMA test illustrated in Fig. 1. They are identified as $0.15,0.145$ and $23,85^{\circ} \mathrm{C}$ to match Young's modulus as shown in Fig. 3. It is observed that the interpolation function is able to accurately predict glassyto-rubbery SMP phase transformation. Next, elastic parameters of $\lambda_{g}, \mu_{g}, \alpha_{g}, \kappa_{g}$ are determined using uniaxial tensile tests and thermal tests as 1.43, 0.615 GPa, $0.00011 / K$, and $1.84 G P a$. Finally, large-strain uniaxial tests presented in Fig. 2 are employed to calibrate parameters of $\psi$, $c_{1 r}, c_{2 r}, Y_{g p}, c_{g f}$ as $1,24,0.55,-0.033 \mathrm{MPa}$ and 1 . Results from the SMP constitutive model are compared with experiments in Fig. 4. It is seen that the model is able to accurately replicate main characteristics observed in the experiments such as low-temperature elasto-plasticity, nearly flat plateau, high-temperature hyper-elasticity and stress-free shape recovery.

As the first example, a tunable reversible unit for bi-stable memory operations is introduced. The tunable mechanical metamaterial unit is designed and fabricated by FDM 3D printing as shown in Fig. 5a. It consists of two curve beams connected to the main body and a movable head. 
Each curved beam has mean radius of $11.55 \mathrm{~mm}, 0.8 \mathrm{~mm}$ thickness, $2 \mathrm{~mm}$ width (depth) and angular span of $90^{\circ}$. The configurations of the unit under loading-unloading process at low temperature, $23{ }^{\circ} \mathrm{C}$, are displayed in Fig. $5 \mathrm{~b}$. It is done in a displacement-control manner. This mechanical loading-unloading process can be considered as a cold programming procedure. The deformed mechanical metamaterial unit is then heated above the transition temperature range to the temperature of $85^{\circ} \mathrm{C}$. It is finally cooled down to $23^{\circ} \mathrm{C}$. Its configuration after heating-cooling process is shown in Fig. 5c. Mechanical response of the unit in terms of force and displacement is presented in Fig. 5e. The FE tool is also implemented to replicate experimental results by simulating one of the curved beam due to the symmetry conditions. A 40×5×1 mesh (40, 5 and 1 elements along radial, thickness and width directions) is used to achieve accurate results converged up to two significant digits. Simulations of are included in Figs. 5d and 5e where $F, W, b$ and $H$ denote vertical force and movement, width and thickness of the 3D printed beam. By applying a compressive mechanical load to the low-temperature unit head, the curved beams are deformed and the unit head moves down as demonstrated in Figs. 5b, 5d and 5e. The unit shows an initial hardening as the force has an increasing trend against the transverse displacement. After a limit point, see Fig. 5e, the unit loses its stability and original shape and shifts to the second stable shape. The unit experiences a snap-through type of buckling. The induced force shows an increasingdecreasing trend and becomes minimum at the end of the snap, so-called the second load limit point, when the unit gets stable at the second mode shape, see Fig. 5b and 5d. After snap-through stage, by further loading, the unit experiences an increasing force in a monotonic manner. By mechanical unloading, it still keeps the second stable shape as shown in Fig. 5b and 5d. It is expected that there would be some plastic strain into the curved beams especially at both ends where maximum stress exits (see Fig. 5d). Once the unit is heated up, it experiences shape recovery 
and gets back to the original stable shape as shown in Figs. 5c, 5d and 5e. Its means the SMP material already had some plastic strain induced by cold programming process and released it simply by heating. Finally, it can be concluded from Fig. 5 that the developed FE tool coupled with the SMP constitutive model is able to simulate experiments very well. It is obseryed that the configuration of the unit arch during loading-unloading-heating-cooling and force-displacement output are replicated accurately.

In the next example, a hot-programming process is applied to the unit. The counterpart of Fig. 5 is illustrated in Fig. 6 for the present problem. The preliminary conclusion drawn from Figs. 5 and 6 is that the high-temperature unit experiences mode shape and force-displacement behavior similar to the low-temperature one. The structure initially in a strain/stress-free state at low temperature of $23{ }^{\circ} \mathrm{C}$ is heated above the transition temperature range, $85{ }^{\circ} \mathrm{C}$. The unit stable at rubbery phase is then loaded mechanically. The results presented in Fig. 6 reveal that the hightemperature unit undergo snap-through buckling phenomenon. It is seen that the force has an increasing-decreasing-increasing trend against the transverse movement. The limit point induced in this test is 300 times lower than that from cold-programming, see Figs. 5e and 6e. This is directly related to the high difference of SMP stiffness at low and high temperatures. The hot metamaterial unit is then cooled down to the low temperature of $23{ }^{\circ} \mathrm{C}$ while its head is being kept fixed. The SMP unit stable at glassy phase is then unloaded by releasing the constraint on the head, see Fig. $6 \mathrm{~b}, 6 \mathrm{~d}$ and $6 \mathrm{e}$. It can be found that the unit does not move significantly by unloading and memorizes a temporary shape. The lack of significant deformation is due to the low level of exciting external force on the glassy-phase unit with much higher stiffness than the rubbery one. In the next step, the low-temperature unit is heated to the temperature of $85{ }^{\circ} \mathrm{C}$ and then cooled back to $23{ }^{\circ} \mathrm{C}$. As it can be seen from the configuration of the unit after heating-cooling process shown in Fig. 6c, 6d 
and 6e, the metamaterial unit can fully recover from the temporary deformed shape to its original shape. It is achieved by releasing the pre-strain produced during the hot programming process. Comparing simulations with experiments in Fig. 6 reveals that the FE tool can accurately predict the unit arch configuration and force-displacement response. The good qualitative and quantitative correlation between experimental and computational results presented in Figs, 5/ and 6 verifies accuracy of the plastic and inelastic strain regimes predicted by the FE tool during cold- and hotprogramming processes. Finally, it is worthwhile to mention that by tuning the temperature of the metamaterial unit, it can be adapted to be programmed in a combination manner of cold and hot programming. Consequently, the force needed for programming can be tuned to meet requirements for desired applications.

The introduced metamaterial units with thermo-mechanical memory operations may have great potentials to mimic electronic memory devices and make surface adaptive structures. For instance, they can be a good candidate to be used for tactile displays. Some researchers utilized piezoelectrics for mimicking tactile Braille characters [27]. The present tunable reversible mechanical metamaterial units could be put on a plane to make a tactile Braille display. The stiffness of every unit could be controlled by customized heating so that each unit could be set to be soft or hard to be moved down. When the surface is rubbed by user's fingers, they could push soft units downward while the hard ones remain unchanged showing the sign. Such mechanical metamaterial units might exhibit another great potential to make shape adaptive surfaces for flow regulation wherever there is a pressure flow like air or fluid pressure. These adaptive units have also potentials for designing adaptive gripper fingers. The set of units could be soft enough to be deformed to grasp the object. Another great potential of this unit is to serve as an active part of a thermo-mechanical valve to close or open a closure. 
If the horizontal constrains of the mechanical metamaterial unit as shown in Fig. 5a are removed, it can be used to design self-deployable bio-medical stents. Fig. 7a shows a 3D printed sub-unit of a stent with self-deploying features. It is composed of four beams connected to movable heads and each other at the ends. Each beam has $15 \mathrm{~mm}$ length, $0.8 \mathrm{~mm}$ thickness, and $2 \mathrm{~mm}$ width (depth) aligned at angle of $70^{\circ}$ with respect to horizon. It is linked to the head and end holder by fillets with mean radius of $2 \mathrm{~mm}$, and angular span of $70^{\circ}$. The configuration of the stent sub-unit under loading-unloading process at low temperature, $23^{\circ} \mathrm{C}$, is demonstrated in Fig. $7 \mathrm{~b}$. Two heads are moved towards each other vertically in a displacement-controlled manner while the other horizontal ends are free. The distance between two heads becomes $4 \mathrm{~mm}$ at the end of loading. By realizing the constraints, the sub-unit deploys partially so that the distance between two heads becomes $18 \mathrm{~mm}$. It is then heated to the temperature of $85^{\circ} \mathrm{C}$ followed by cooling down to $23^{\circ} \mathrm{C}$. The stent sub-unit configuration after heating-cooling process is illustrated in Fig. 7c. Fig. 7e also shows force-displacement behavior of the sub-unit during loading-unloading and shape recovery. Computational results from the FE simulations of one of the curved beams are also included in Fig. 7d and 7e where $F, W, b$ and $H$ denote/vertical force and movement with respect to the $X$ axis, width and thickness of the 3D printed beam. A 50×5×1 mesh (50, 5 and 1 elements along length, thickness and width directions) is used to achieve accurate results converged up to two significant digits. As it can be seen, the stent sub-unit experiences a linear force-displacement response at the beginning of the mechanical loading. However, beyond $W / H=2$, the mechanical metamaterial response softens undergoing a nearly flat plateau, see Fig. 7e. This is due to the growth of plastic strain at end of beams where there is high level of stress. Once the constraints are removed, the sub-unit partially recovers its original shape as shown in Fig. 7b, 7d and 7e. The residual plastic deformation leads the sub-unit to keep up to around $50 \%$ of the original height. The cold- 
programmed stent could be placed inside a catheter and then delivered to a desired location for deployment purpose. Once the stent is pushed out, it may be heated by blood temperature and mechanically self-deploys by fully shape recovery, see Figs. $7 \mathrm{c}, 7 \mathrm{~d}$ and $7 \mathrm{e}$. It can serve as a supporting function for opening the artery keeping blood flowing normally. Finally, comparative studies reveal that the structural/material model and solution method are capable of replicating the experiments with excellent accuracy.

The next example is dedicated to demonstrate potential applications of cold programming for introducing rolling-up chiral mechanical metamaterial. Fig. 8a displays a 3D printed four-beam chiral structure fixed on a frame. The central annular ring with $4.6 \mathrm{~mm}$ mean radius, $1.6 \mathrm{~mm}$ thickness and $2 \mathrm{~mm}$ width is held by four SMP beams. Each beam has $20 \mathrm{~mm}$ length, $0.8 \mathrm{~mm}$ thickness, and $2 \mathrm{~mm}$ width (depth) tangential to the annular ring at each quarter. A $50 \times 5 \times 1$ mesh (50, 5 and 1 elements along length, thickness and width directions) for each beam and a $30 \times 10 \times 1$ mesh (30, 10 and 1 elements along circumferential, thickness and width directions) for the ring are used to achieve accurate results converged up to two significant digits. The frame is stretched transversely to get $6.4 \mathrm{~mm}$ displacement. The frame is then unloaded mechanically. The experimental and computational configuration of the chiral metamaterial after loading-unloading stage is depicted in Fig. 8b. The force-displacement response of the chiral metamaterial during loading-unloading is also depicted in Fig. 8d. Parameters of $F, W, b$ and $H$ denote vertical force and movement with respect to the $X$ axis, width and thickness of the 3D printed beams. As it can be seen, stretching the structure leads the annular ring to rotate. A quasi bi-linear hardening behavior is observed in Fig. 8d. It implies that the mechanical metamaterial rotates first with an almost linear force-displacement and then resists to rotation and becomes locked showing overall linear hardening. After unloading, it keeps a turn as much as 30 degrees. This would be due to the 
growth of plastic strains at the ends of the beams via the cold-programming process. In the next stage, the chiral metamaterial is heated from $23{ }^{\circ} \mathrm{C}$ to $85^{\circ} \mathrm{C}$ and then cooled back to $23^{\circ} \mathrm{C}$. Fig. $8 \mathrm{c}$ and $8 \mathrm{~d}$ show the configuration after heating-cooling and thermal shape recovery path, respectively. It can be found that the rolling-up feature is reversible as the mechanical metamaterial recovers its initial shape upon heating. Beyond the metamaterial reversible translation, the current design allows to implement more sophisticated functions such as this reversible rotation.

As a final example, a mechanical metamaterial structure with supportive function is introduced and investigated. An auxetic is printed as shown in Fig. 9a. It has horizontal beams with $20 \mathrm{~mm}$ mean length which are linked with oblique members with $10 \mathrm{~mm}$ mean length aligned at angle of $70^{\circ}$ with respect to the horizon. All members have $0.8 \mathrm{~mm}$ thickness, and $10 \mathrm{~mm}$ width (depth). $50 \times 5 \times 1$ and $30 \times 5 \times 1$ meshes are used for the horizontal and oblique beams to achieve accurate results converged up to two significant digits. The lattice is fixed on a frame to facilitate mechanical testing. The auxetic is well-known to have a negative Poisson's ratio in the elastic domain. It is first compressed transversely by an Aluminum cylinder with external diameter of 55 $\mathrm{mm}$. It is compressed by $15.6 \mathrm{~mm}$ transverse movement, see Fig. 9b. Then the auxetic experiences unloading and 23-85-23 ${ }^{\circ} \mathrm{C}$ heating-cooling processes as illustrated in Fig. 9c and 9d. The forcedisplacement curve of the auxetic mechanical metamaterial during loading-unloading and thermal shape recovery are also depicted in Fig. 9e where $F, W, b$ and $H$ denote vertical force and movement with respect to the horizon, width and thickness of the $3 \mathrm{D}$ printed beams. It is seen from Fig. $9 \mathrm{~b}$ that the structure shrinks in the axial and transverse directions by loading in the transverse direction. When the external object hits the metamaterial and compresses it in one direction, the material contracts laterally as well due to the internal axial compression. In fact, the material flows into the location of collision creating a denser structure which is higher resistant to collision. This 


\section{Conclusion}

This paper was aimed at introducing tunable multi-stable metamaterials with reversible shape memory operations by 4D FDM printing technology. Thermo-mechanical behaviors of SMPs were experimentally characterized revealing elasto-plastic and hyper-elastic responses at glassy and rubbery phases. Cold and hot programming concepts were combined with structural characteristics to design and fabricate adaptive mechanical metamaterials with reversible shape memory features. This was achieved by developing a 3D macroscopic constitutive model and implementing geometrically non-linear FEM. The governing equations were solved by an elastic-predictor plastic-corrector return mapping approach based on the iterative Newton-Raphson and Riks techniques to trace non-linear equilibrium path in the finite strain regime. The large-strain material-structural model with accurate capability of simulating phase transformation, hyperelasticity, elasto-plasticity and hot-cold programming was utilized to design and print mechanical metamaterials. The feasibility and performance of the tunable reversible metamaterial unit with 
bi-stable memory operations were assessed experimentally and numerically. They were considered as potential candidates in mimicking electronic memory devices like tactile displays and designing surface adaptive structures. Different design of the unit combined with cold programming exhibited another great potential to serve as parts of self-deployable bio-medical stents. Furthermore, experiments were conducted to demonstrate potential applications of cold programming for fabricating recoverable rolling-up chiral mechanical metamaterials and loadresistance supportive auxetics. The material-structural formulation, concepts and results provided in this paper are expected to be instrumental towards printing tunable adaptive metamaterials with reversible shape memory operations.

\section{Acknowledgments}

The work described in this paper was supported by the Research Grants Council of the Hong Kong Special Administrative Region, China (Project No. CUHK 14202016) and Vice-Chancellor's Discretionary Fund / CUHK T Stone Robotics Institute (Project ID: 4930762).

\section{References}

[1] Kadic M, Buckmann T, Stenger N, Thiel M and Wegener M 2012 On the practicability of pentamode mechanical metamaterials Appl Phys. Lett. 100191901

[2] Ren X, Shen J, Ghaedizadeh A, Tian H and Xie Y M 2015 Experiments and parametric studies on 3D metallic auxetic metamaterials with tuneable mechanical properties Smart Mater. Struct. 24(9) p.095016

[3] Ranjbar M, Boldrin L, Scarpa F, Neild S and Patsias S 2016 Vibroacoustic optimization of anti-tetrachiral and auxetic hexagonal sandwich panels with gradient geometry Smart Mater. Struct. 25(5) p.054012

[4] Wang Y, Zhao W, Wang H and Liu Z 2018 A bio-inspired novel active elastic component based on negative Poisson's ratio structure and dielectric elastomer Smart Mater. Struct. Article in Press DOI: doi.org/10.1088/1361-665X/aaea22 
[5] Ren X, Das R, Tran P, Ngo T D and Xie Y M 2018 Auxetic metamaterials and structures: A review Smart Mater. Struct. 27(2) p.023001

[6] Mizzi L, Mahdi E M, Titov K, Gatt R, Attard D, Evans K E, Grima J N and Tan J C 2018 Mechanical metamaterials with star-shaped pores exhibiting negative and zero Poisson's ratio Mater. Des. 146 28-37

[7] Ren X, Shen J, Ghaedizadeh A, Tian H and Xie Y M 2016 A simple auxetic tubular structure with tuneable mechanical properties Smart Mater. Struct. 25 p. 065012

[8] Li T, Chen Y, Hu X, Li Y and Wang L 2018 Exploiting negative Poisson's ratio to design 3Dprinted composites with enhanced mechanical properties Mater. Des. 142 247-258

[9] Bodaghi M, Damanpack A R, Hu G F and Liao W H 2017 Large deformations of soft metamaterials fabricated by 3D printing Mater. De.s 131 81-91

[10] Chen Y, Li T, Scarpa F and Wang L 2017 Lattice metamaterials with mechanically tunable Poisson's ratio for vibration control Phys Rev. Appl. 7(2) p.024012

[11] Hedayati R, Mirzaali M J, Vergani L and Zadpoor A A 2018 Action-at-a-distance metamaterials: Distributed local actuation through far-field global forces APL Mater 6(3) p.036101

[12] Haghpanah B, Salari-Sharif L, Pourrajab P, Hopkins J and Valdevit L 2016 Multistable shapereconfigurable architected materials Adv Mater. 28(36) 7915-7920

[13] Rafsanjani A, Akbarzadeh A and Pasini D 2015 Snapping mechanical metamaterials under tension Adv Mater. 27(39) 5931-5935

[14] Che K, Yuan C, Wu J, Qi H J and Meaud J 2016 3D-printedmultistablemechanical metamaterials with a deterministic deformation sequence J. Appl Mech. 84011004

[15] Chen C C, Ishikawa A, Tang Y H, Shiao M H, Tsai D P and Tanaka T 2015 Uniaxial-isotropic metamaterials by three-dimensional split-ring resonators Adv Optical. Mater. 3 (1) 44-48

[16] Kwok T H, Wang C C L, Deng D, Zhang Y and Chen Y 2015 4D printing for freeform surfaces: design optimization of origami and kirigami structures Trans ASME J. Mech. Des. 137111712

[17] Bodaghi M, Damanpack A R and Liao W H 2016 Self-expanding/shrinking structures by 4D printing Smart Mater. Struct. 25 p.105034

[18] Mitchell A, Lafont U, Hołyńska M and Semprimoschnig C 2018 Additive manufacturing-a review of 4D printing and future applications Add Manufacture. 24 606-626

[19] Bodaghi M, Damanpack A R and Liao W H 2017 Adaptive metamaterials by functionally graded 4D printing Mater. Des. 135 26-36

[20] Bodaghi M, Damanpack A R and Liao W H 2018 Triple shape memory polymers by 4D printing Smart Mater. Struct. 27(6) p.065010

[21] Ding Z, Weeger O, Qi H J and Dunn M L 20184D rods: 3D structures via programmable 1D composite rods Mater. De.s 137 256-265

[22] Akbari S, Sakhaei A H, Kowsari K, Yang B, Serjouei A, Yuanfang Z and Ge Q 2018 Enhanced multimaterial/4D printing with active hinges Smart Mater. Struct. 27(6) p.065027

[23] ASTM D638-102010 Standard Test Method for Tensile Properties of Plastics 1-16

[24] Truesdell C and Noll W 1965 The Non-Linear Field Theories of Mechanics (Berlin: Springer)

[25] Simo J C and Hughes T J R 1998 Computational Inelasticity New York: Springer

[26] Reddy J N 2004 An Introduction to Nonlinear Finite Element Analysis New York: Oxford University Press Inc. 


\section{List of Figures}

Fig. 1. DMA test on the 3D printed SMP: (a) Young and storage moduli; (b) $\tan (\delta$ ).

Fig. 2. Large-strain response of the 3D printed SMP under uniaxial loading-unloading at 23 (a) and $85^{\circ} \mathrm{C}$ (b) (Green dashed line shows stress-free strain recovery by thermal loading).

Fig. 3. Young's modulus from DMA test and trigonometric interpolation function.

Fig. 4. Comparison between model predictions and experimental results for uniaxial loading-unloading of the 3D printed SMPs at 23 (a) and $85^{\circ} \mathrm{C}$ (b) (Green dashed line shows stress-free strain recovery by thermal loading).

Fig. 5. Tunable metamaterial unit under cold-programming: experimental (a)-(c) and computational (d) configuration (color bar shows axial force $(\mathrm{N} / \mathrm{m})$ along the length direction); (e) equilibrium path for loading-unloading and thermal shape recovery (Green dashed line shows stress-free strain recovery by thermal loading).

Fig. 6. Tunable metamaterial unit under hot-programming: experimental (a)-(c) and computational (d) configuration (color bar shows axial force $(\mathrm{N} / \mathrm{m})$ along the length direction); equilibrium path for loading in hot-programming and thermal shape recovery (square and diamond markers show experimental and numerical results at the end of cooling-unloading; green dashed line shows stress-free strain recovery by thermal loading).

Fig. 7. Stent sub-unit under cold-programming: experimental (a)-(c) and computational (d) configuration (color bar shows axial force $(\mathrm{N} / \mathrm{m})$ along the length direction); (e) equilibrium path for loading-unloading and thermal shape recovery (Green dashed line shows stress-free strain recovery by thermal loading).

Fig. 8. Rolling-up chiral metamaterial under cold-programming: (a)-(c) experimental and computational configuration (red line is representative of simulated deformation mid-surface patterns); (d) equilibrium path for loading-unloading and thermal shape recovery (Green dashed line shows stress-free strain recovery by thermal loading),

Fig. 9. Supportive auxetic metamaterial under cold-programming: (a)-(d) experimental and computational configuration (red line is representative of simulated deformation mid-surface patterns); (e) equilibrium path for loading-unloading and thermal shape recovery (Green dashed line shows stress-free strain recovery by thermal loading). 
(a)

(b)
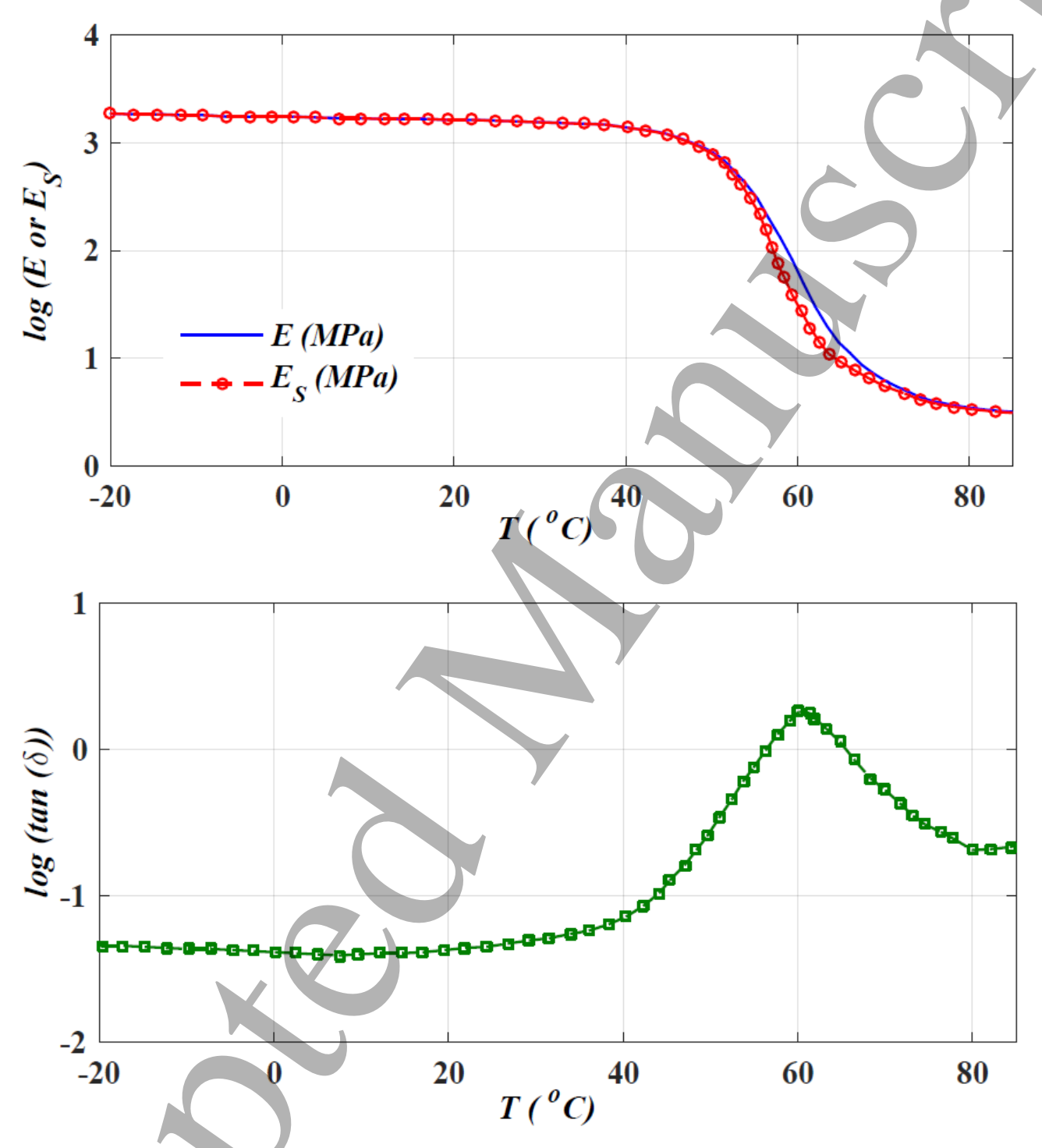

Fig. 1. DMA test on the 3D printed SMP: (a) Young and storage moduli; (b) $\tan (\delta$ ) 
(a)

(b)
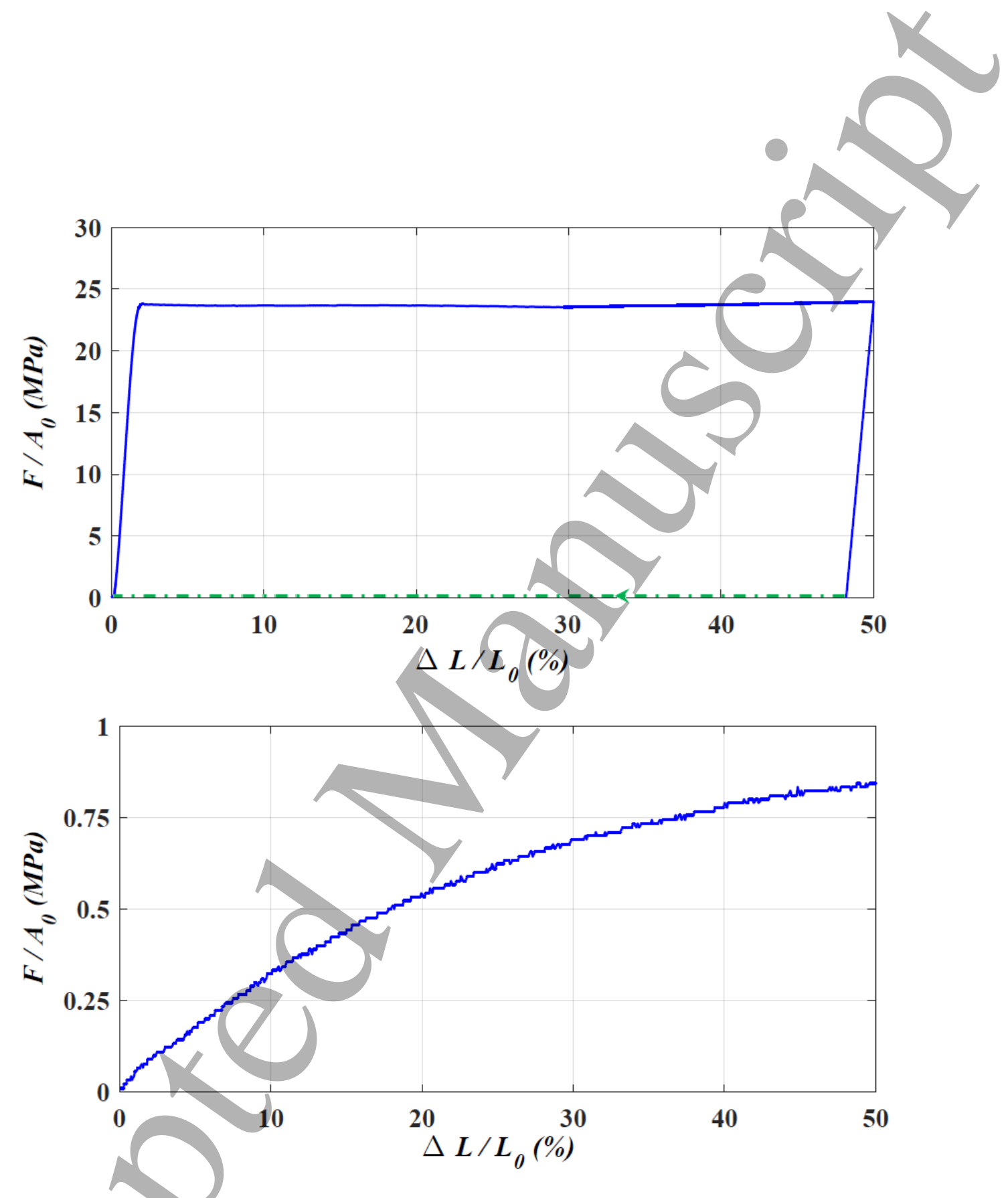

Fig. 2. Large-strain response of the 3D printed SMP under uniaxial loading-unloading at 23 (a) and 85 ${ }^{\circ} \mathrm{C}$ (b) (Green dashed line shows stress-free strain recovery by thermal loading). 


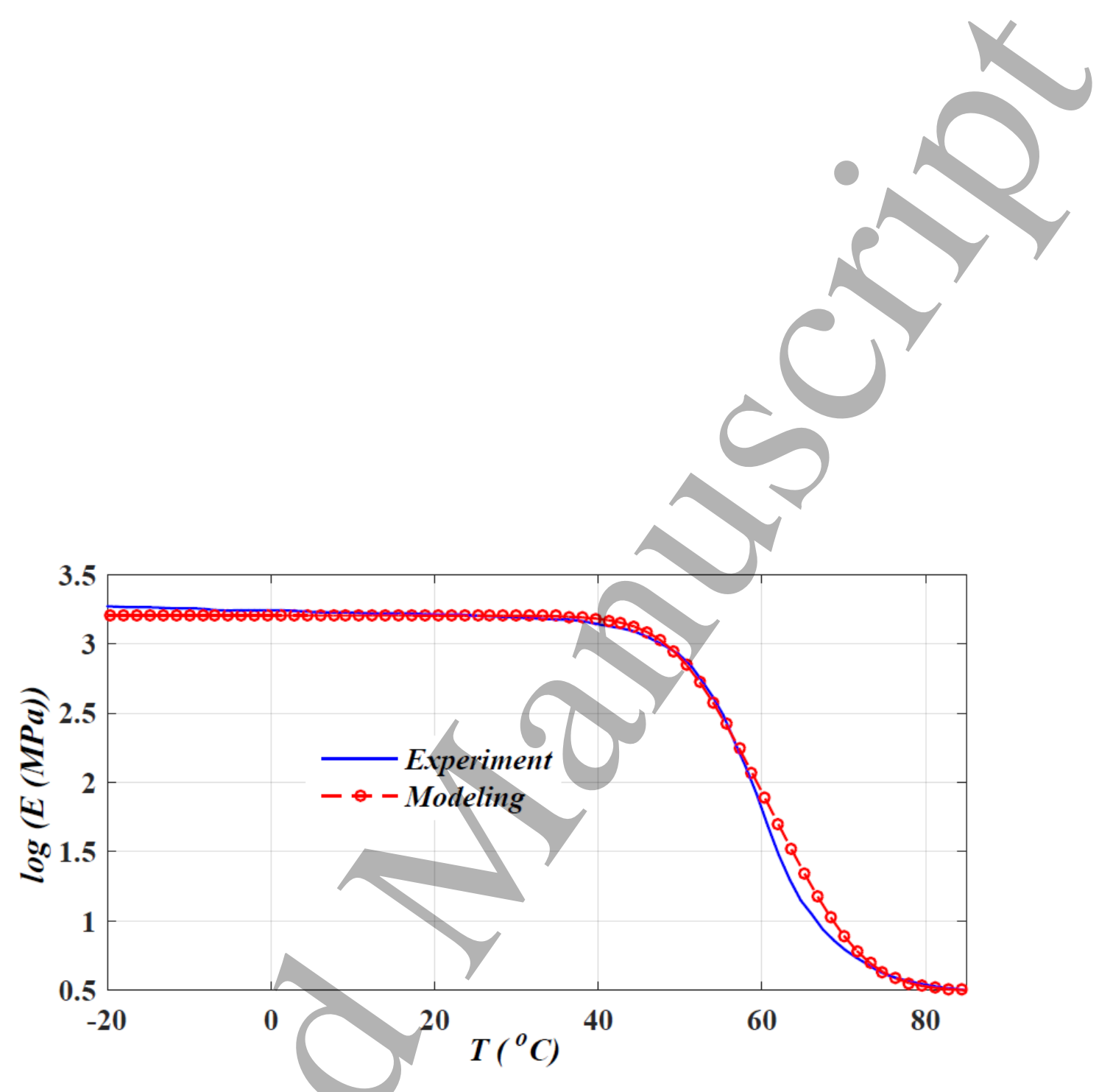

Fig. 3. Young's modulus from DMA test and trigonometric interpolation function.

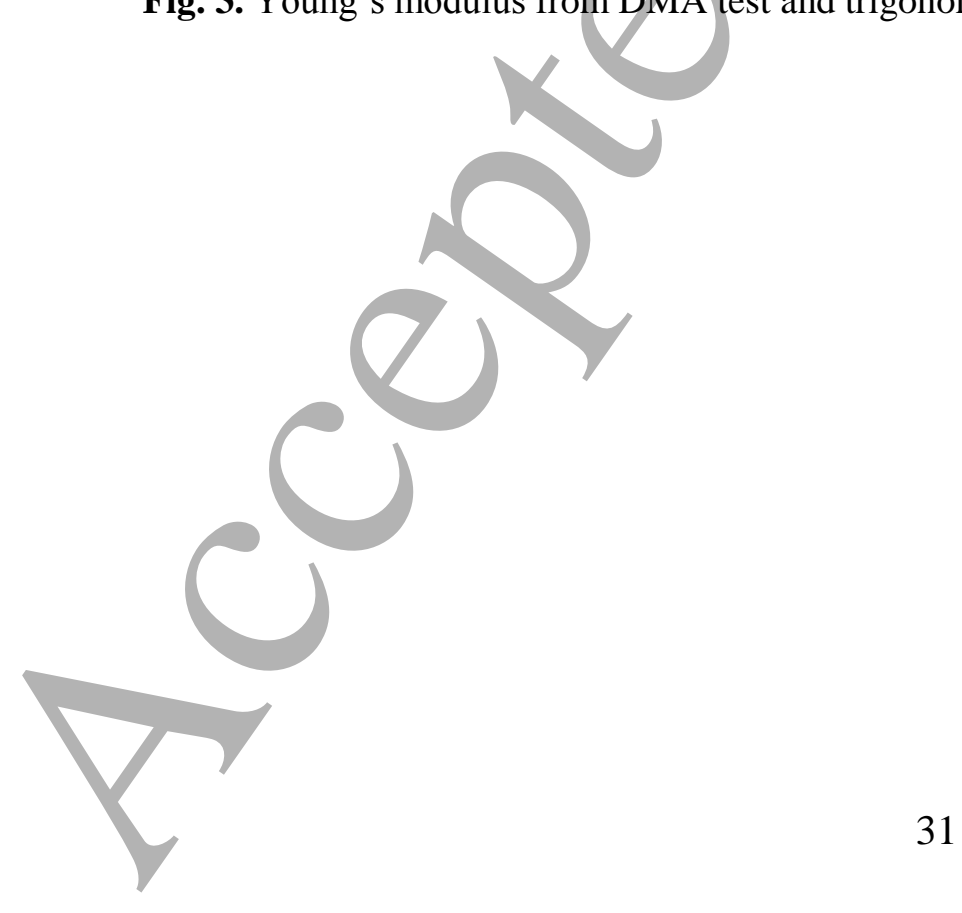


(a)

(b)
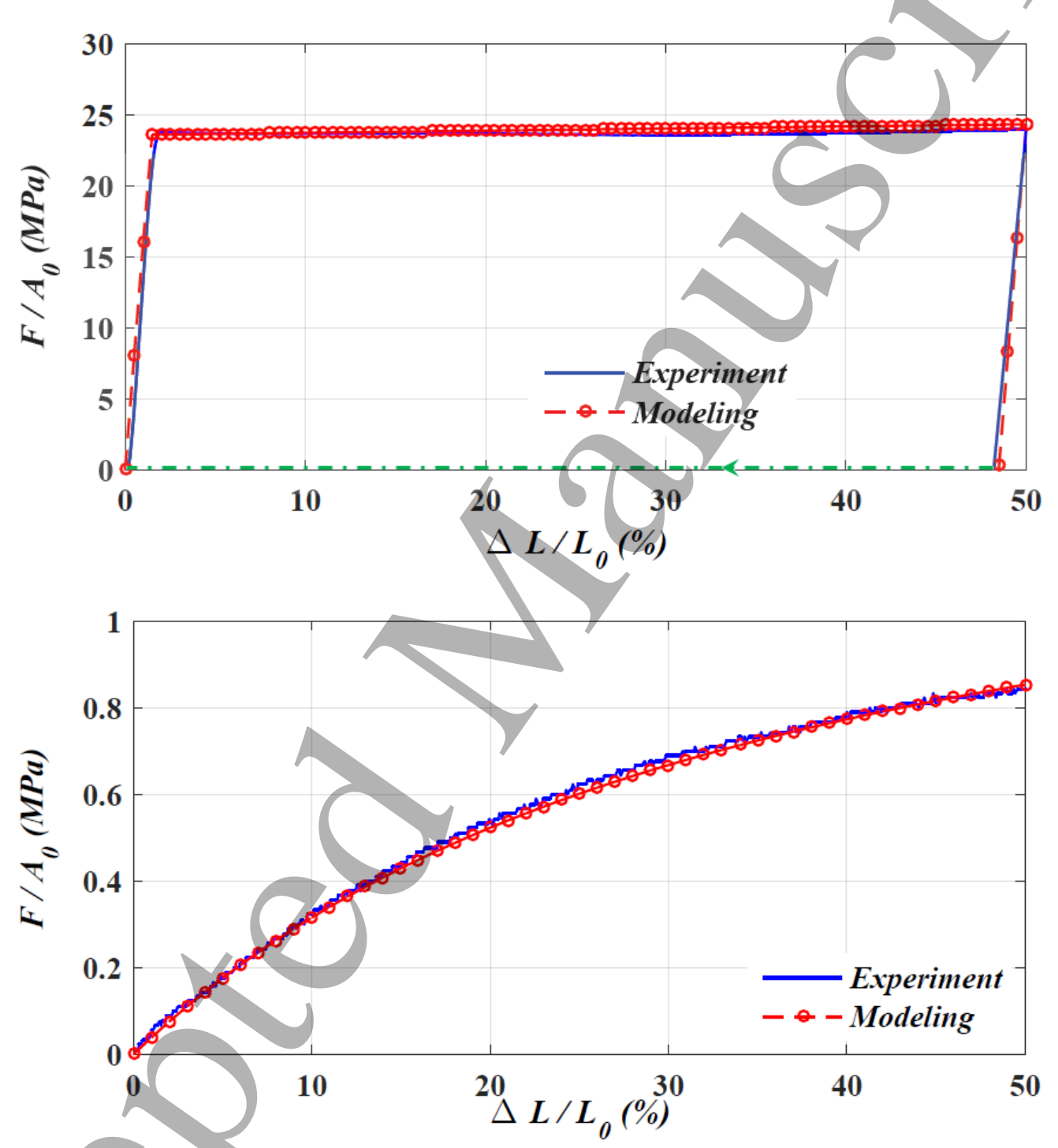

Fig. 4. Comparison between model predictions and experimental results for uniaxial loading-unloading of the 3D printed SMPs at 23 (a) and $85^{\circ} \mathrm{C}$ (b) (Green dashed line shows stress-free strain recovery by thermal loading). 
(a)

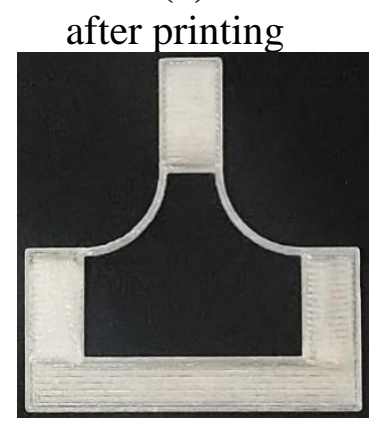

(b)

end of loading-unloading

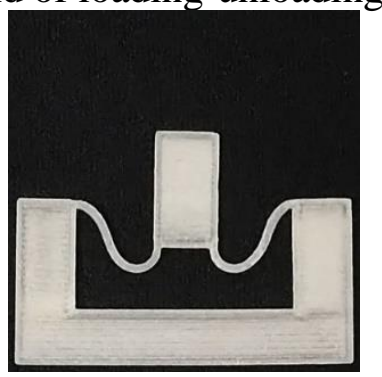

(d) (c) end of heating-cooling

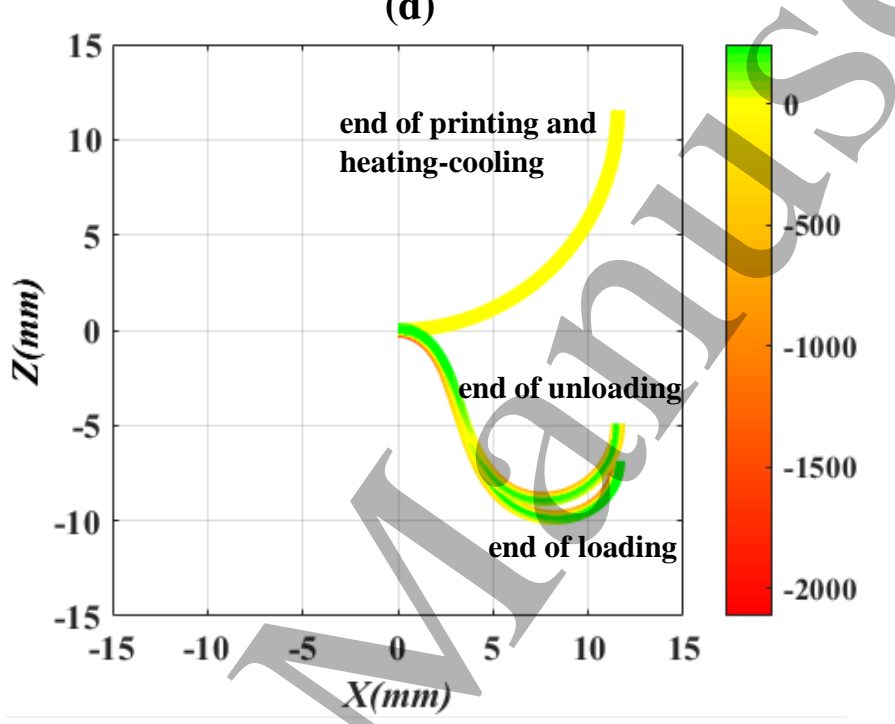

(e)

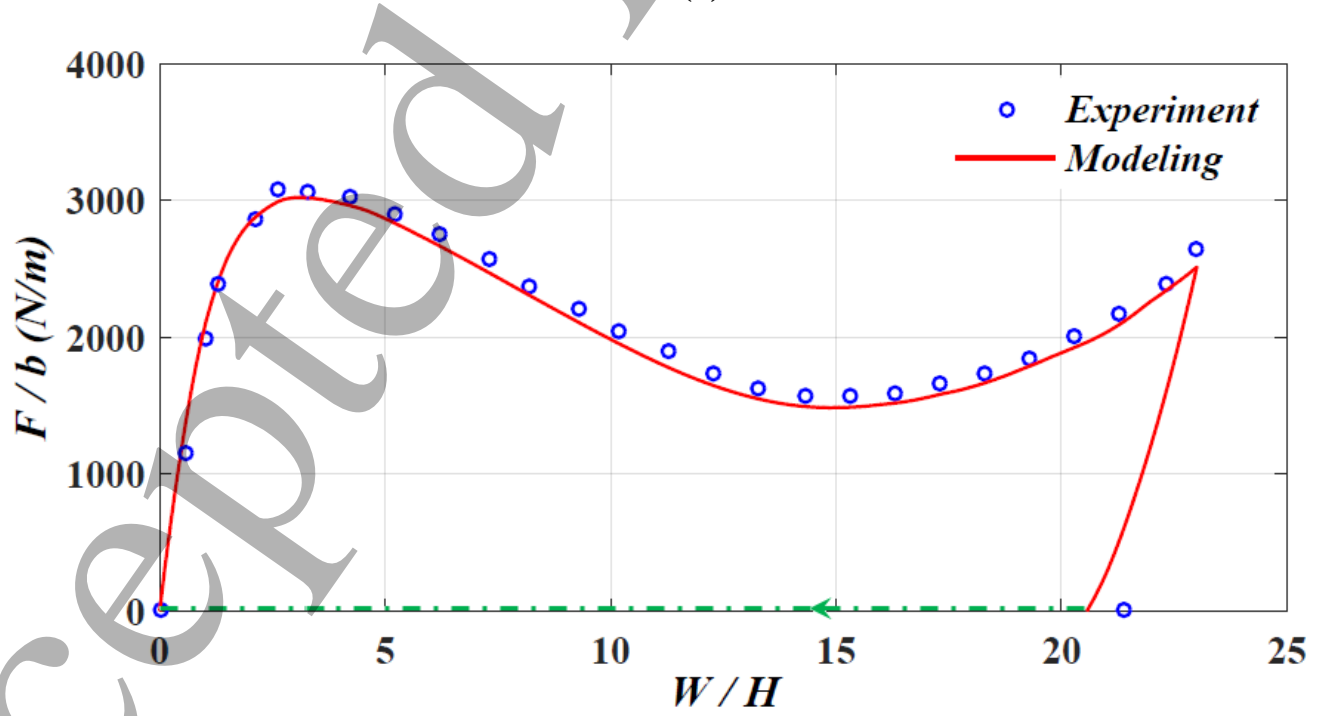

Fig. 5. Tunable metamaterial unit under cold-programming: experimental (a)-(c) and computational (d) configuration (color bar shows axial force $(\mathrm{N} / \mathrm{m})$ along the length direction); (e) equilibrium path for loading-unloading and thermal shape recovery (Green dashed line shows stress-free strain recovery by thermal loading). 
(a)

after printing

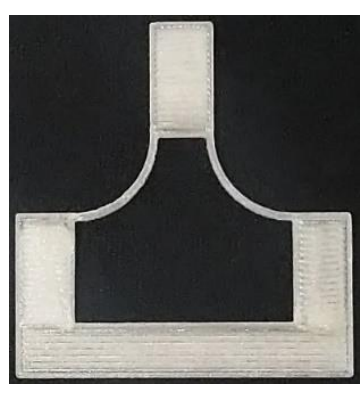

(b)

end of loading-coolingunloading

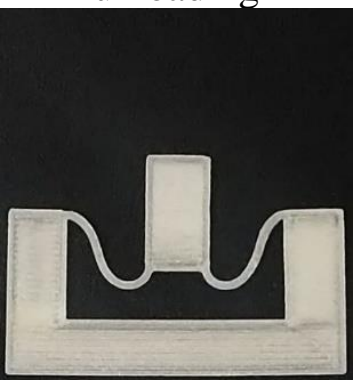

(d)

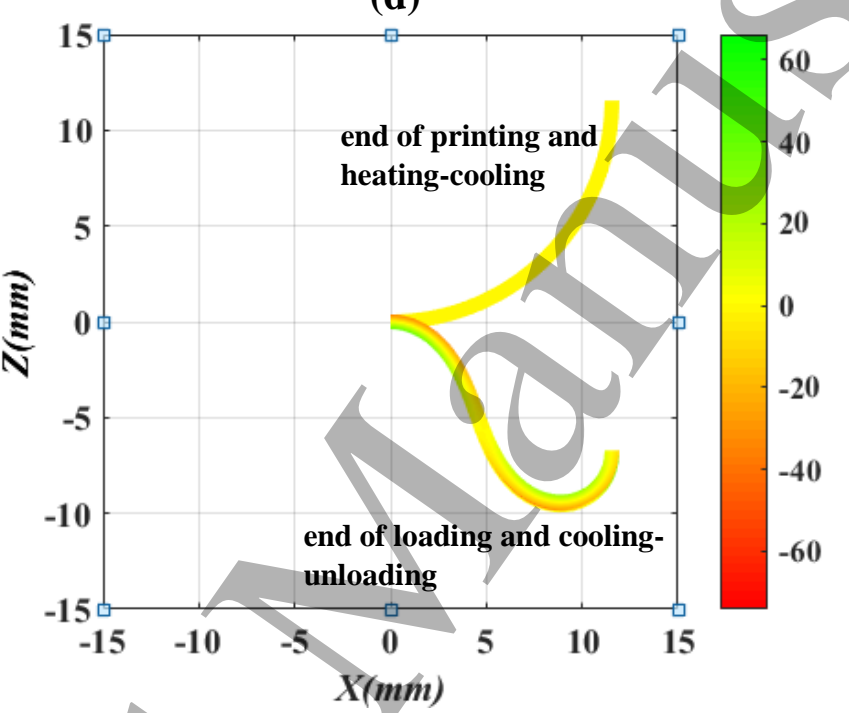

(e)

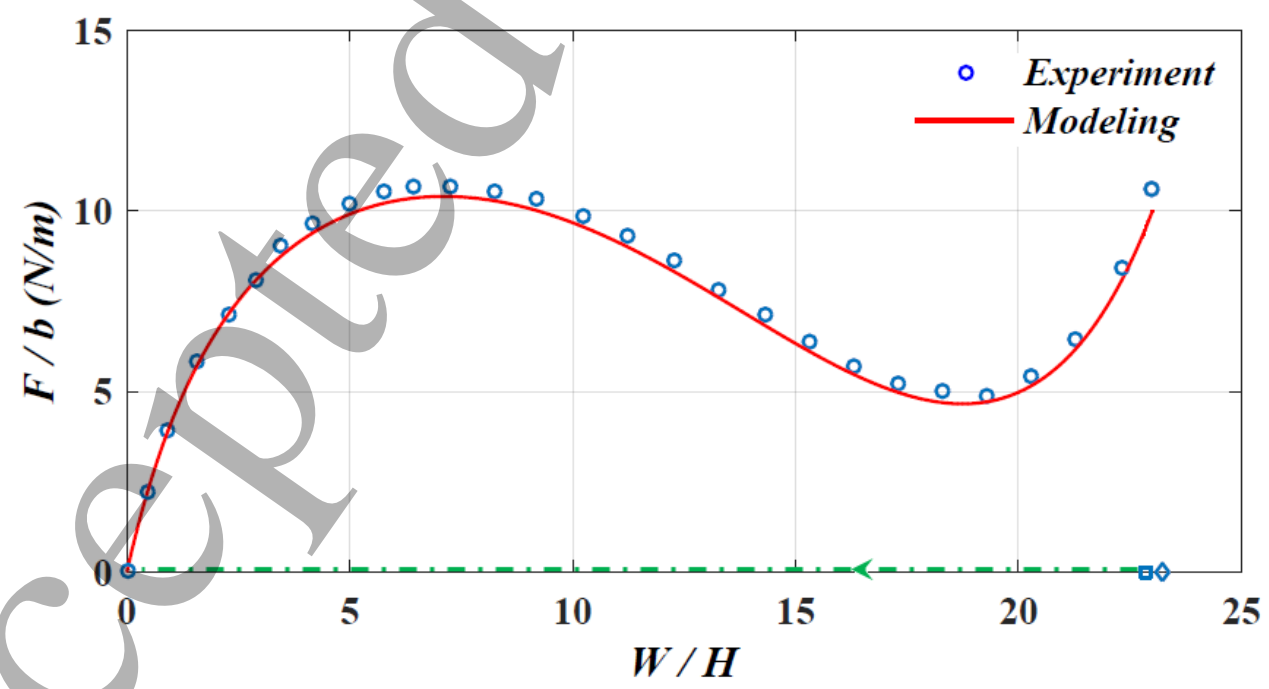

Fig. 6. Tunable metamaterial unit under hot-programming: experimental (a)-(c) and computational (d) configuration (color bar shows axial force $(\mathrm{N} / \mathrm{m})$ along the length direction); equilibrium path for loading in hot-programming and thermal shape recovery (square and diamond markers show experimental and numerical results at the end of cooling-unloading; green dashed line shows stress-free strain recovery by thermalloading). 
(a)

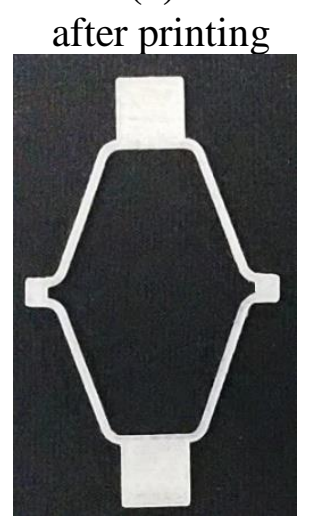

(b)

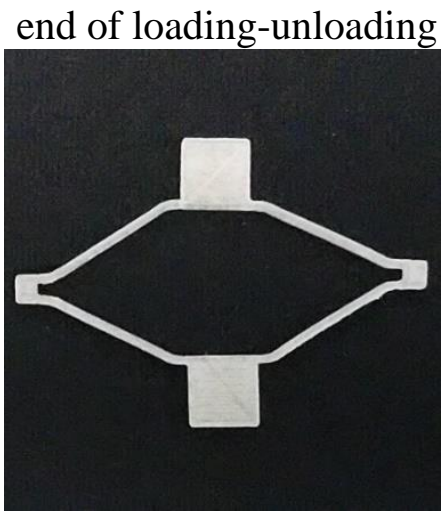

(d)

(e)

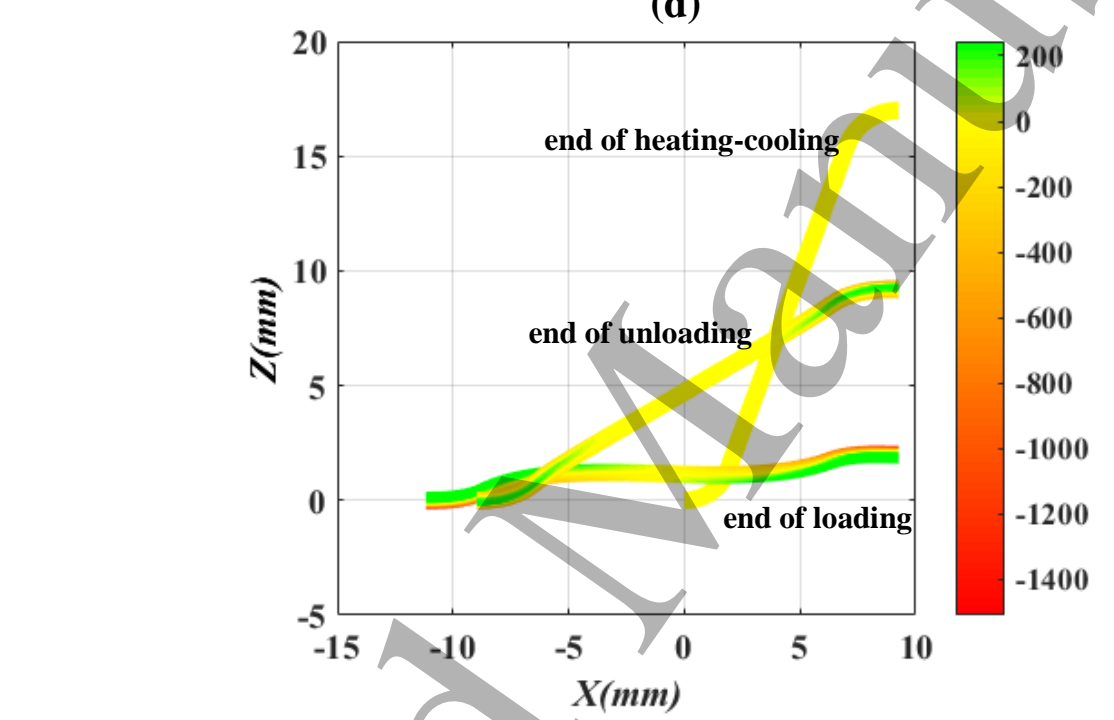

(c)

end of heating-cooling

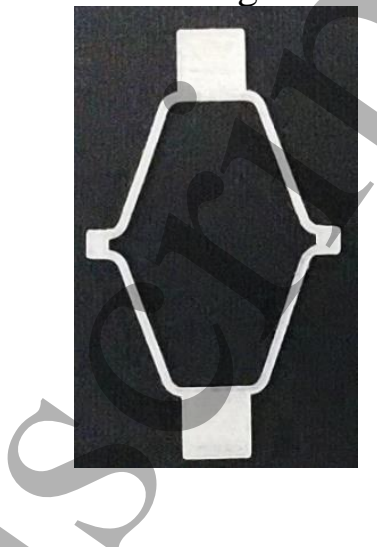

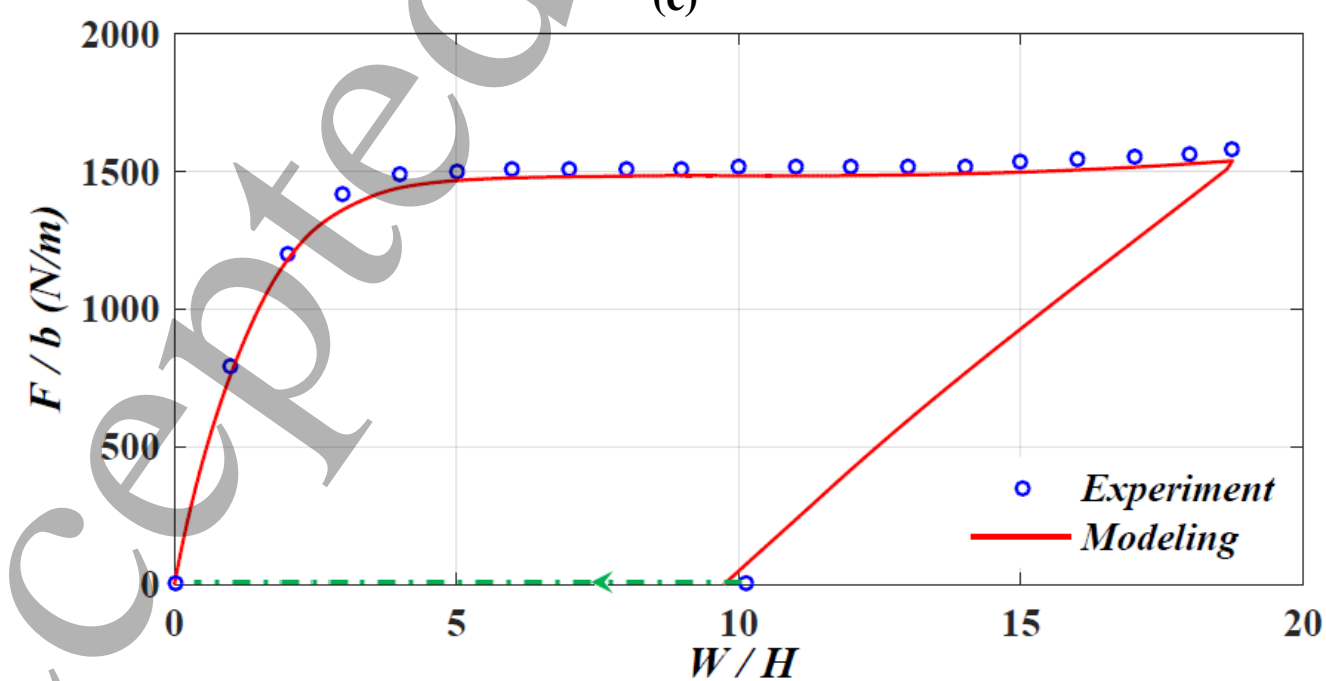

Fig. 7. Stent sub-unit under cold-programming: experimental (a)-(c) and computational (d) configuration (color bar shows axial force $(\mathrm{N} / \mathrm{m})$ along the length direction); (e) equilibrium path for loading-unloading and thermal shape recovery (Green dashed line shows stress-free strain recovery by thermal loading). 
(a)

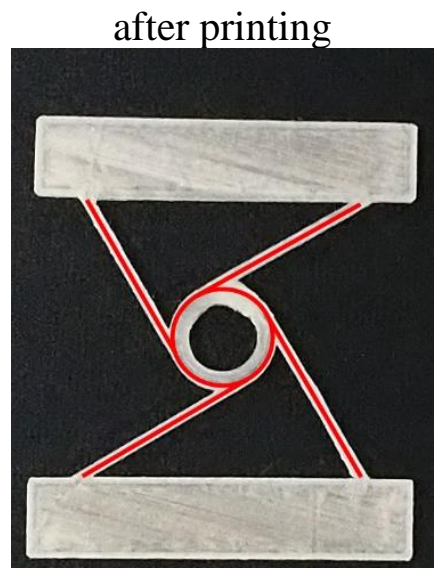

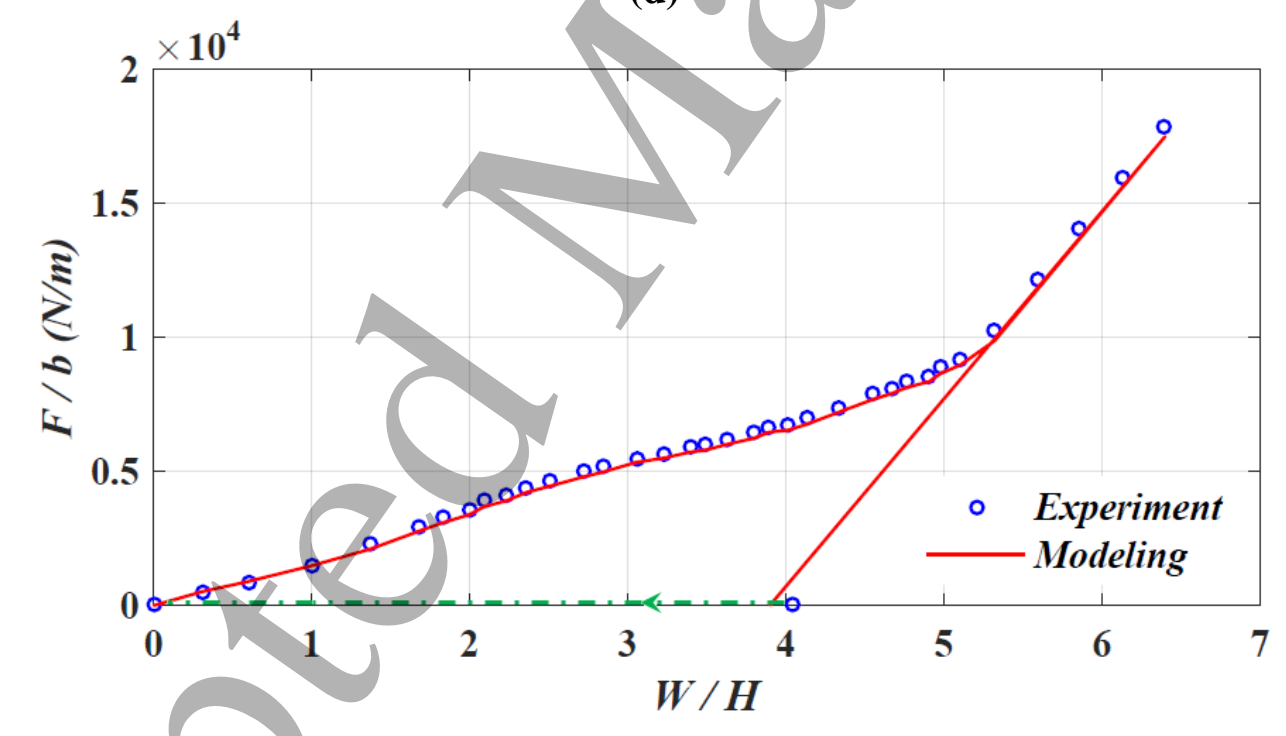

(b)

end of loading-unloading

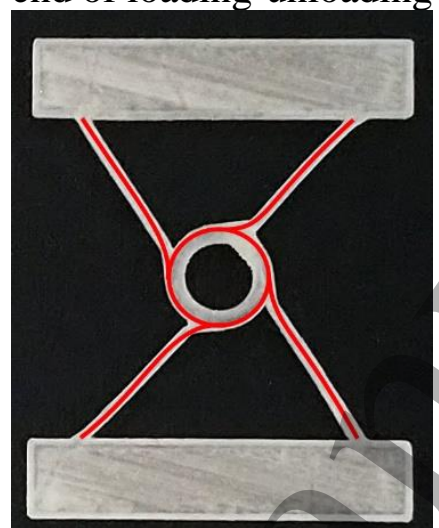

(d) (c)

end of heating-cooling

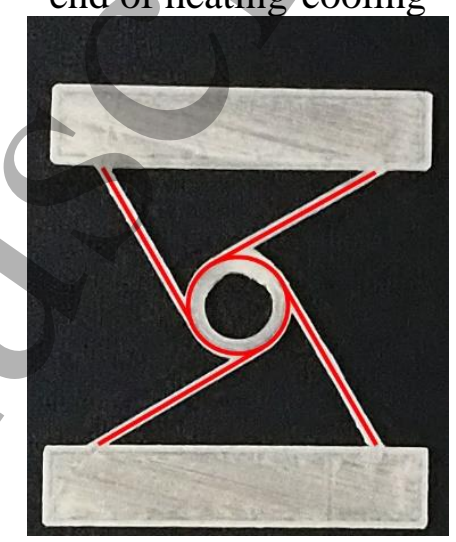

(d)

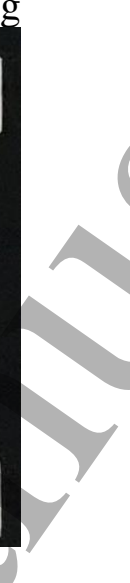

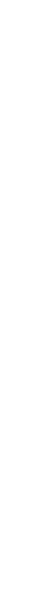

Fig. 8. Rolling-up chiral metamaterial under cold-programming: (a)-(c) experimental and computational configuration (red line is representative of simulated deformation mid-surface patterns); (d) equilibrium path for loading-unloading and thermal shape recovery (Green dashed line shows stress-free strain recovery by thermal loading). 
(a)

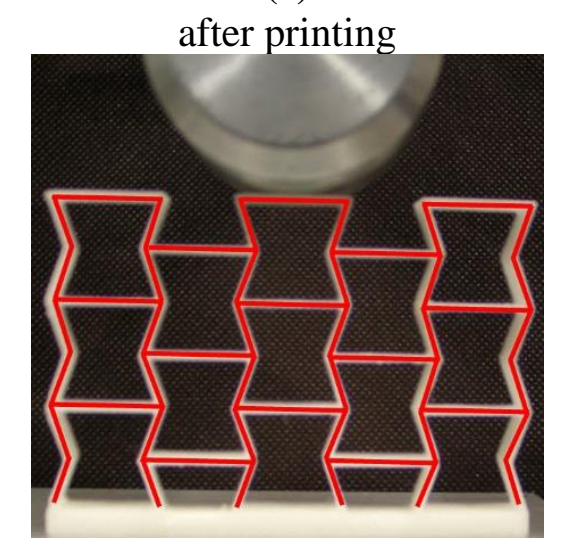

(c)
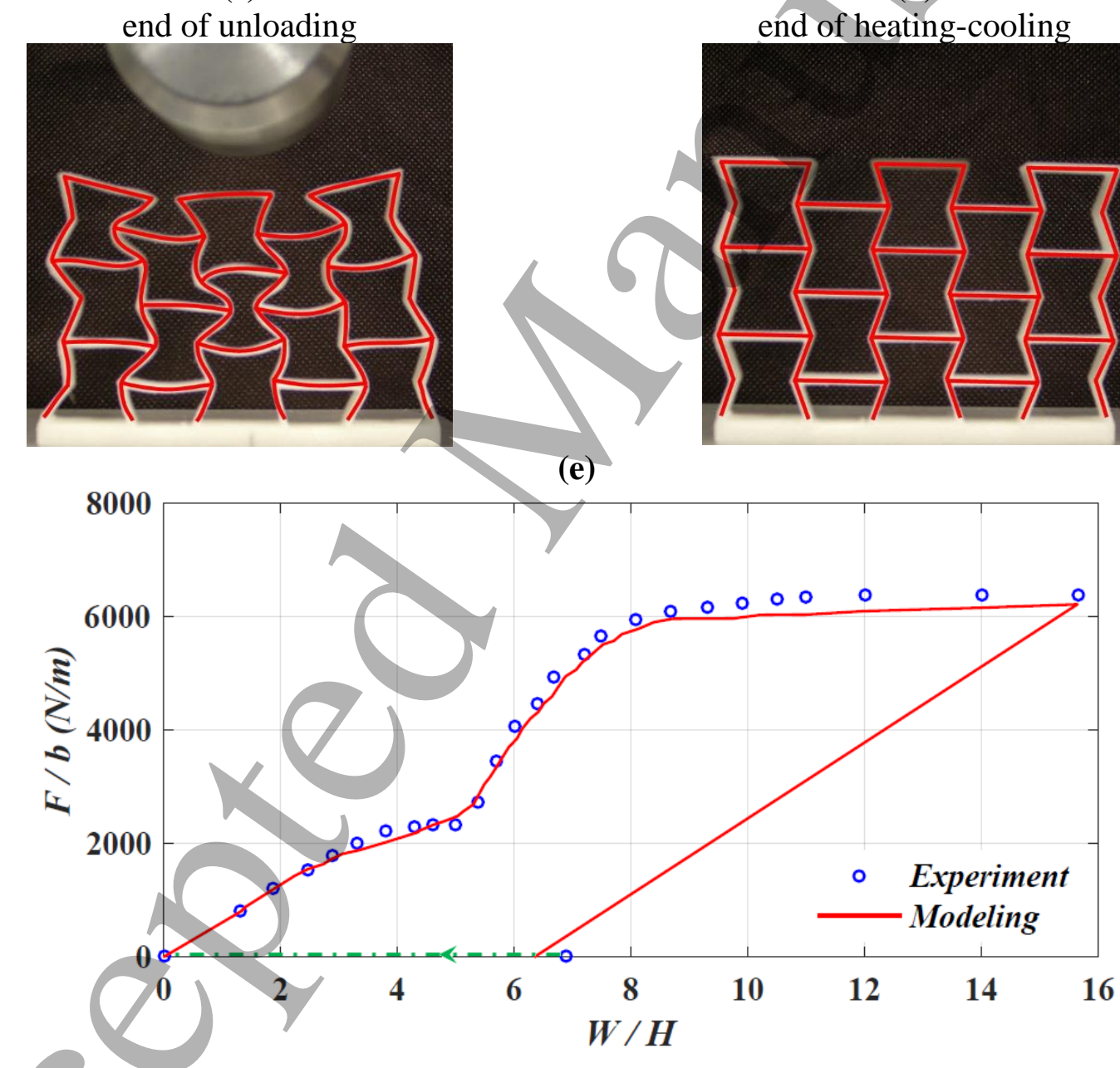

(b)

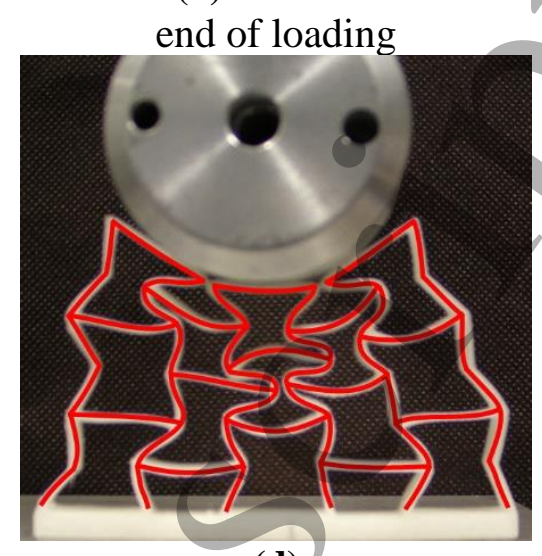

(d)

end of heating-cooling

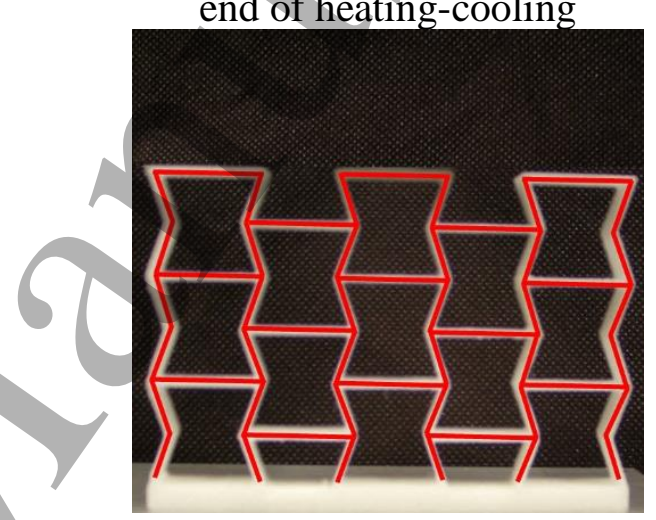

e)

Fig. 9. Supportive auxetic metamaterial under cold-programming: (a)-(d) experimental and computational configuration (red line is representative of simulated deformation mid-surface patterns); (e) equilibrium path for loading-unloading and thermal shape recovery (Green dashed line shows stressfree strain recovery by thermal loading). 